\title{
Territorio y crisis: impacto territorial de la crisis económica en las regiones metropolitanas de Madrid y Barcelona
}

\author{
Miguel Rubiales Pérez \\ Departament de Geografia \\ Universitat de Barcelona \\ mrubiale@gmail.com
}

Territorio y crisis: impacto territorial de la crisis económica en las regiones metropolitanas de Madrid y Barcelona (Resumen)

La crisis económica ha tenido un gran impacto en el país, pero ha sido escasamente estudiada en su dimensión socioterritorial. Para analizar desde esta óptica su impacto en las regiones metropolitanas de Barcelona y de Madrid, 1) se establece una secuenciación temporal de las tasas de paro; 2) se analiza la evolución temporal de las tasas de paro según las principales características sociodemográficas de la población: edad, sexo y nacionalidad; y también, 3) en función de sus características ocupacionales: sector de actividad y categoría profesional. Después, 4) se compara la evolución de las tasas de paro en diferentes agregados territoriales; para finalmente 5) se lanzan análisis multivariable para caracterizar los componentes estructurales de las tasas de desempleo en cada periodo y región. Este análisis muestra que la crisis tiene dos fases distintas, que incide antes y más intensamente en las periferias, en las zonas más vinculadas a las actividades constructivas y en los municipios donde más se concentra la población más vulnerable.

Palabras clave: Crisis, Región metropolitana, paro registrado, clases sociales, desempleo territorial.

Crisis and urban territory: spatial impact of the economic crisis in the metropolitan areas of Madrid and Barcelona (abstract)

The economic crisis has had a major impact on the country, but has been little studied in its socio-territorial dimension. To analyze it in the metropolitan areas of Barcelona and Madrid, we 1) present a time series of the unemployment rates, the evolution of which will be 2) analyzed against the main sociodemographic features of the studied population: age, gender and nationality. 3) These results are further broken down by sector of activity and professional category to account for the occupational characteristics of said population. 4) Next, the obtained time series of unemployment rates are analyzed from a geographical perspective, through the comparison of territorial aggregates such as the cluster of municipalities by size, 
by comarcas (NUTS4), and by socioeconomic level. Finally, 5) the evolution of said unemployment rates for each period and metropolitan region is studied through multivariate analysis. The obtained results allow for the presentation of a synthesis of the processes that underlie the changes in unemployment rates and their territorial articulation. This analysis shows that the crisis has two distinct phases, with an earlier and more intense incidence in the regional peripheries, in areas most linked to the construction activities and in the municipalities where the most vulnerable populations concentrate.

Keywords: Crisis, metropolitan region, registered unemployment, social classes, territorial unemployment.

El objetivo del análisis que se realiza aquí es doble. En primer lugar, dar cuenta del impacto socioterritorial de la crisis económica en la Región Metropolitana de Barcelona y en la Región Metropolitana de Madrid (RMB y RMM en adelante). En segundo lugar, comprobar hasta qué punto las diferencias socioterritoriales y de clase social se encuentran relacionadas con una mayor o menor incidencia de la crisis ${ }^{1}$.

En principio, cabría esperar que los territorios metropolitanos más afectados por la crisis sean aquellos que se encuentran en una posición subordinada bien en función de su ubicación periférica, bien a causa de las características socioeconómicas de su población residente y su mercado laboral local. Además, es una buena oportunidad para comprobar si la crisis atenúa o acelera la tendencia que, durante los años 2000, tenían las capitales globales a reforzar su posición respecto al resto del territorio nacional $^{2}$. Finalmente, el presente análisis es una buena oportunidad para comprobar las diferencias entre los periodos y verificar hasta qué punto puede comprobarse la hipótesis de una crisis extendida o de dos crisis casi consecutivas pero esencialmente distintas.

Para analizar el impacto de la crisis y medir su alcance desde una perspectiva cuantitativa, una dimensión clave es el trabajo. El uso de datos de desempleo como medida operativa del alcance de la crisis, responde a esta centralidad social y estadística del trabajo. El trabajo resulta fundamental en la articulación de identidades sociales y en la construcción de la ciudadanía ${ }^{3}$. No sólo eso, para muchos hogares es la única fuente de recursos con los que satisfacer aquellas necesidades básicas que no tienen un acceso universal garantizado. Además, resulta necesario basar este análisis en los registros de desempleo porque son la única fuente que permite relacionar, a escala municipal, el deterioro económico general y sus efectos en la vida de las personas en función de su municipio, características sociodemográficas y ocupacionales.

\footnotetext{
${ }^{1}$ Este trabajo forma parte del proyecto de I+D "Desigualdad social, polarización territorial y formación de espacios vulnerables en las grandes áreas metropolitanas españolas” (CSO2015-65219-C2-1-R), dirigido por Isabel Pujadas y Fernando Gil, y financiado por el Ministerio de Economía y Competitividad a través del Plan Estatal de Investigación Científica y Técnica y de Innovación 20132016 (MINECO/FEDER, UE). El autor forma parte del grupo de investigación consolidado "Territorio, Población y Ciudadanía”, reconocido por la Generalitat de Cataluña (GRC_2014SGR380).

${ }^{2}$ Graham y Marvin, 2001.

${ }^{3}$ Alonso, 2006.
} 


\section{Metodología}

El análisis del impacto territorial de la crisis en función del desempleo registrado en la RMB y la RMM requiere ciertas precisiones sobre el ámbito de estudio, las fuentes de datos explotadas y algunas precisiones técnicas sobre los análisis estadísticos aplicados. A parte de estas precisiones, en cada apartado se irá ilustrando el funcionamiento del análisis desarrollado.

\section{Ámbito de estudio}

Las regiones metropolitanas de Madrid y Barcelona suponen el 24,4\% de la población del estado, conjuntamente llegan a producir el 31\% del PIB de España (datos provisionales 2008), son las regiones más interconectadas en las redes de la economía global y el primer (o principal) lugar de residencia de las elites y clases altas establecidas en nuestro país. A partir de los años ochenta, Madrid y Barcelona, junto con sus respectivas regiones metropolitanas, han sufrido procesos paralelos de desconcentración poblacional y redistribución de su población en base a migraciones internas. Las respectivas cabeceras se suman al ritmo de crecimiento de sus regiones metropolitanas de la mano del flujo de migraciones internacionales, especialmente intenso durante el periodo 2001-2007. Este ciclo terminó recientemente, con la primera fase de la crisis iniciada en 2008 e incluso comienza a mostrar los primeros signos de retroceso.

Las dos áreas analizadas presentan diferencias importantes. En primer lugar en relación a su consolidación administrativa. La Región Metropolitana de Barcelona (RMB) es uno de los siete ámbitos definidos en el Pla Territorial General de Catalunya, con una definición que se remonta a la década de los sesenta ${ }^{4}$, y se configura en numerosos estudios como la unidad de análisis metropolitana preferente. La Región Metropolitana de Madrid (RMM) es, frecuentemente aproximada a los límites administrativos de la Comunidad Autónoma de Madrid (CAM), empleando este territorio como ámbito metropolitano de referencia. La RMB, ocupa una superficie menor, de unos $3.200 \mathrm{~km}^{2}$ englobando 164 municipios que suponen, aproximadamente, la mitad de la provincia de Barcelona, mientras que la CAM incluye 178 municipios y una extensión de $8.038 \mathrm{~km}^{2}$. Respecto al tamaño poblacional, la provincia de Madrid contaba casi con un millón más de habitantes que la provincia de Barcelona (y con más de 1.460 .000 habitantes más que la RMB) según el padrón de 2011; finalmente, el análisis de ambas regiones es levemente diferente (en la forma de calcular algunas de sus tasas) debido que aún no se dispone de la información de afiliados a la Seguridad Social en Cataluña con el suficiente detalle.

\section{Fuentes de datos}

Los datos estadísticos elaborados a partir de los registros de desempleo constituyen el eje de análisis de este artículo. Cuentan con varias ventajas, como su universalidad, su actualización anual y cierto nivel de desagregación territorial. Ofrecen información completa sobre el número de personas inscritas como desempleados: municipio de residencia, sexo, edad, nacionalidad, sector de la ocupación anterior y categoría

\footnotetext{
${ }^{4}$ Esteban, 2003.
} 
profesional de la ocupación anterior, pero adolecen de algunos problemas metodológicos:

1) En primer lugar, los sesgos derivados de la naturaleza de la fuente. Los registros administrativos tienden a infravalorar el número de desempleados. La voluntariedad de inscripción en este registro y la falta de incentivos para producirla, lleva a procesos de autoselección. No toda la población que se encuentra desempleada se registra en los servicios públicos de empleo. Los jóvenes, en su primera incorporación al mercado laboral, tienden a no incluir el registro en los servicios de empleo públicos como parte de su búsqueda activa de empleo. Los trabajadores irregulares, sin derecho a prestación, tienden a no inscribirse cuando pierden el empleo. La limitación temporal del tiempo prestación, hasta un máximo de dos años, y las renovaciones de registro que se deben realizar para no causar baja automática, producen cada año un porcentaje de desistimiento que se conoce como el efecto desánimo. Son trabajadores que dejan de estar registrados como demandantes de empleo sin haber encontrado trabajo. En tiempos de crisis prolongada y paro estructural este efecto aumenta.

2) En segundo lugar, esta fuente no tiene una población de referencia sobre la que calcular las tasas de desempleo, paso imprescindible para poder comparar la evolución de municipios y agrupaciones de diferente tamaño poblacional. Las poblaciones de referencia que pueden utilizarse son: la población potencialmente activa, de 16 a 64 años (Padrón Municipal), y la población ocupada (con los registros de afiliación a la Seguridad Social. Esta fuente aún no se encuentra disponible para la RMB con la suficiente desagregación).

\section{Análisis multivariable}

Los análisis factoriales de componentes principales realizados sobre el cambio en las tasas de desempleo de los municipios de la RMB y la RMM cubren una gran parte de la variabilidad de los datos y cumplen satisfactoriamente con los criterios de validez y

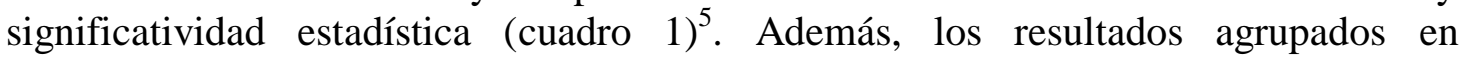
conglomerados muestran bastante consistencia teórica y territorial. En el apartado 6, se explica de forma didáctica el funcionamiento de este análisis y de su combinación con el análisis de conglomerados.

Tres fuentes de ruido podrían dificultar la obtención resultados: los sesgos del paro registrado, calcular tasas a partir de dos fuentes de datos diferentes (que, por ejemplo, clasifican las ocupaciones siguiendo criterios distintos) y la heterogeneidad de las unidades administrativas empleadas para agrupar la información (municipios). Para evitarlo, se han llevado a cabo algunas precauciones. Se analiza la variación de tasas

\footnotetext{
${ }^{5}$ La prueba de esfericidad de Barlet, la adecuación muestral de Kaiser-Meyer-Olkin y el porcentaje de varianza explicada son las medidas más empleadas para medir la adecuación de los datos al análisis factorial desde el punto de vista estadístico. La prueba de esfericidad calcula la probabilidad de que las correlaciones del conjunto de variables formen una matriz identidad (lo que implica que no estén relacionadas entre sí y que el análisis no se puede realizar). La adecuación muestral KMO mide el nivel de correlación entre los valores de variables diferentes, y por ende de "bondad" o utilidad del análisis factorial. Finalmente, el porcentaje de varianza explicado calcula hasta qué punto el resumen que realiza el análisis factorial recoge o pierde parte de la información inicial.
} 
en periodos cortos y comparables (cada uno de 2 años), cuando se producen los cambios más intensos (2008-2010 y 2011-2013). Al trabajar sobre el cambio relativo de tasas, algunas categorías de las variables presentaban valores absolutos bajos para los que pequeñas variaciones en números absolutos presentaban tasas de cambio muy grandes. Para minimizar los efectos derivados del uso de estas tasas, se han agrupado en restos comarcales a los municipios con una población menor de 5.000 habitantes.

Cuadro 1

Resultados de los análisis factoriales realizados

\begin{tabular}{|c|c|c|c|}
\hline \multirow[b]{2}{*}{ Factorial } & \multicolumn{3}{|c|}{ Criterios de validez y significatividad } \\
\hline & $\begin{array}{c}\text { Significatividad } \\
\text { esfericidad de Bartlett }\end{array}$ & $\begin{array}{c}\% \text { explicado de la } \\
\text { varianza }\end{array}$ & $\begin{array}{l}\text { adecuación muestral } \\
\text { de Kaiser-Meyer-Olkin }\end{array}$ \\
\hline RMB 2010 & 0,000 & 79,9 & 0,65 \\
\hline RMB 2013 & 0,000 & 72,1 & 0,60 \\
\hline RMM 2010 & 0,000 & 86,0 & 0,72 \\
\hline RMM2013 & 0,000 & 77,7 & 0,63 \\
\hline
\end{tabular}

\section{El paro registrado, serie temporal de la crisis}

A modo introductorio, se compara la evolución del desempleo en distintas fuentes y escalas, así como el saldo migratorio. El objetivo es establecer los periodos de análisis en función de la variación uniforme del desempleo y comprobar hasta qué punto distintas fuentes y procesos presentan variaciones congruentes durante la crisis. Así, esta comparación permite establecer unos periodos de análisis en función de los momentos de variación intensa del desempleo. En el gráfico de la figura 1, puede observarse que tanto las tasas de paro de la EPA, como las tasas del paro registrado, detectan una importante crisis de empleo de 2008 a 2010, la originada a raíz del colapso financiero internacional y del sector de la construcción. En ambas fuentes, se aprecia la relativa estabilización en el empleo que produjeron las políticas de inversión pública activadas entre 2009 y 2010, así como el segundo periodo de crisis caracterizado por los recortes en servicios, control del gasto público y política fiscal contractiva que se introducen a mitad de 2010 y muestran sus efectos a partir de 2011.

La EPA y el paro registrado son también congruentes con los cambios relacionados pero externos al mercado laboral, como las variaciones de los saldos migratorios totales. En todo caso, la evolución general presenta dos periodos de aumentos marcados de las tasas de desempleo (2008-2010 y 2011-2013) y acredita la capacidad de los registros de desempleo como fuente capaz de dar cuenta de las grandes alteraciones que ha sufrido el mercado laboral.

En este gráfico se incluyen las tasas de paro registrado de la RMM calculadas sobre el total de población potencialmente activa. Este mismo cálculo de tasas de desempleo sobre ocupados no puede hacerse la RMB porque los registros de la Seguridad Social no están aún disponibles. En las figuras 1 y 2 se aprecia que los niveles de desempleo en la RMM no suben tanto durante la primera fase de la crisis, pero luego aceleran durante la segunda, llegando a resultados similares a los que alcanza la RMB. 
Figura 1

Evolución de las tasas de paro y del saldo migratorio según diferentes ámbitos y fuentes

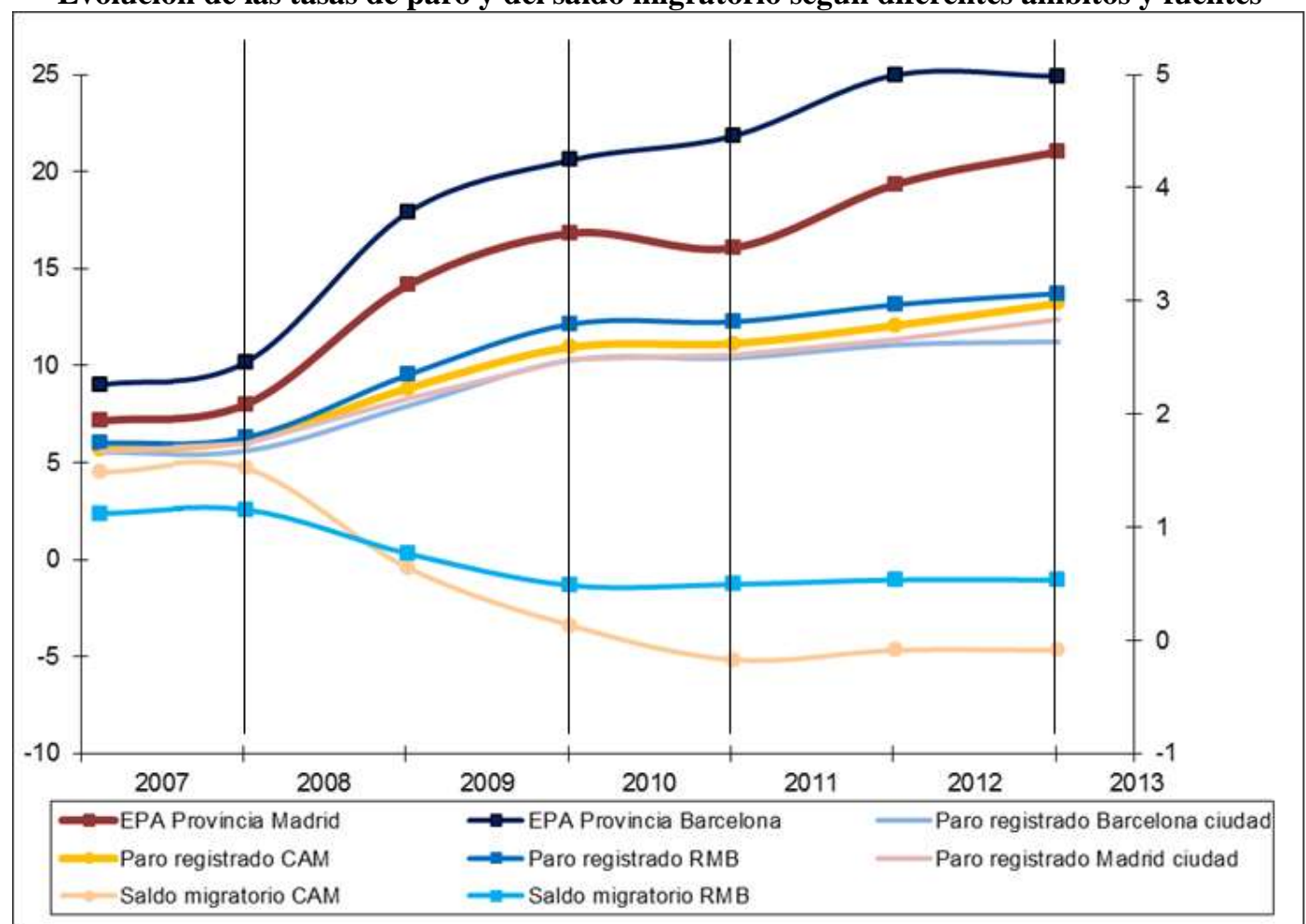

Fuente: Instituto de Estadística de Madrid, Observatori Metropolità del Treball, registros de desempleo; INE, tasas de desempleo de la Encuesta de Población Activa EPA y Padrón Continuo. Los valores de 2013 se han estimado a partir de la población de 2012.

\section{Transformaciones en las tasas de desempleo registrado en función de las principales variables demográficas}

En este apartado, así como en el 4 y en el 5, se hace un seguimiento de la evolución de las tasas de desempleo en función de las variables sociodemográficas, ocupacionales y territoriales. El objetivo es doble: por una parte identificar características y elementos concretos de evolución de la crisis en la RMB y RMM; por otra, ir presentando y comprendiendo las variables que formarán parte del análisis multivariable del apartado 6.

\section{El desempleo por edades}

A grandes rasgos, la evolución de las tasas de desempleo brutas por grupos de edad reproduce el esquema temporal de dos periodos de aumento que se analizó en el apartado 4. Para comparar la evolución en función de los diferentes grupos de edad, las tasas se calculan para el grupo de jóvenes adultos y para los trabajadores mayores de 45 años.

Se han excluido de los cálculos los grupos de menos edad por su comportamiento atípico. Por las mismas razones, en el gráfico de la figura 2 las tasas de la RMM están calculadas sobre el total de ocupados, lo que produce niveles superiores a los de la 
RMB (calculados sobre el total de la población), pero mantiene la relación entre los grupos de edad.

Figura 2

Evolución de la proporción de paro registrado según edad (población y ocupados RMM)

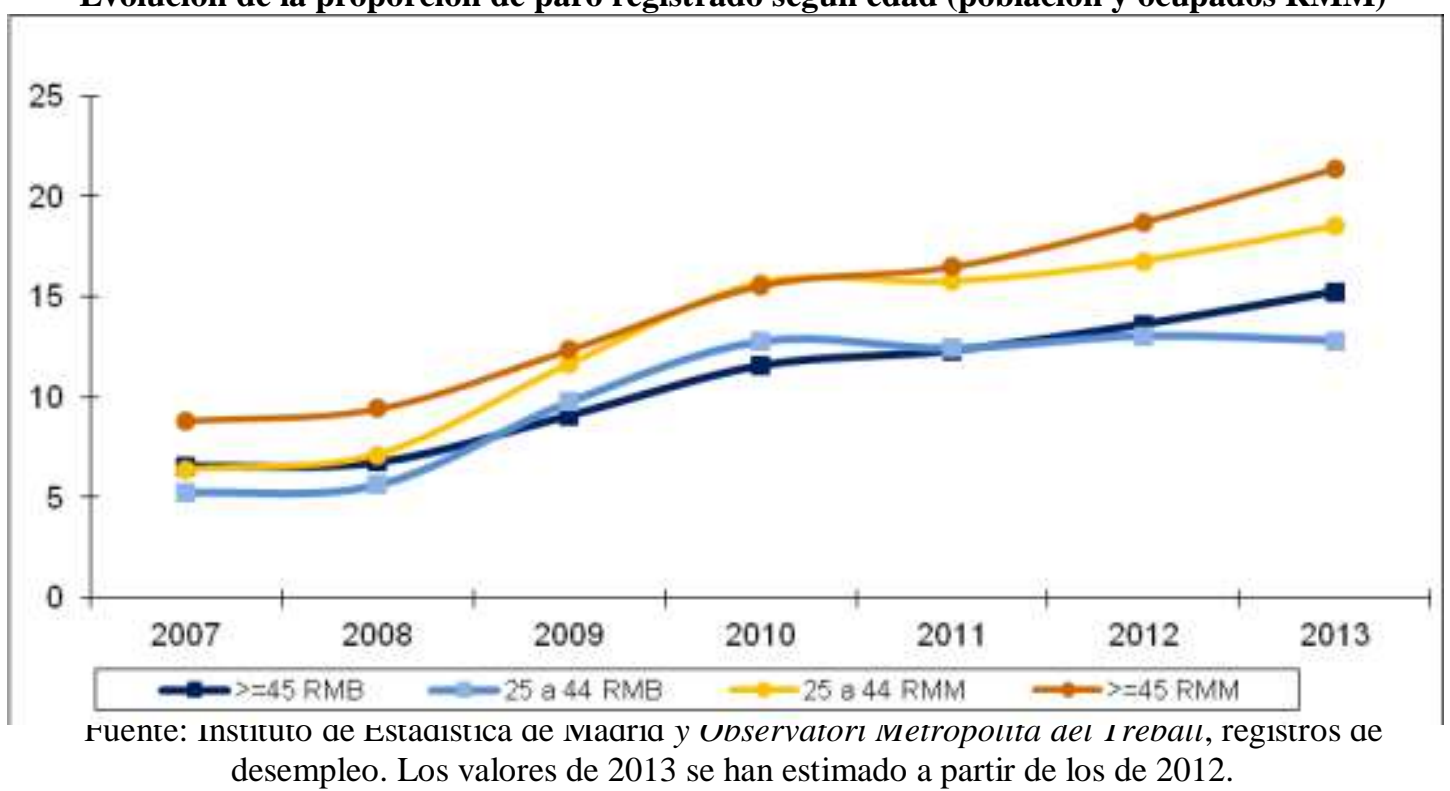

A partir de la segunda fase de la crisis (2011), el crecimiento de las tasas de desempleo del grupo de trabajadores de más de 45 años se acelera en comparación con el de los trabajadores más jóvenes. Hay que destacar que, aunque la primera aceleración del desempleo para los trabajadores de más edad coincide con el estallido de la crisis (2008), en esa fase es el grupo de edad que menos empeora. Sin embargo, a partir de 2010 sus tasas de desempleo mantienen un alto ritmo de crecimiento (figura 2).

Este comportamiento del desempleo de los trabajadores mayores apunta un proceso que va más allá del cierre de empresas tradicionales con plantillas envejecidas. Parece que, independientemente de la evolución de su actividad, algunas empresas aprovechan el nuevo clima laboral y social para renovar plantillas sustituyendo los trabajadores mayores por otros de menor edad y coste. Un análisis sobre la jubilación de los trabajadores mayores ${ }^{6}$ muestra cómo durante la crisis de 1993-1994, se produjo una reducción de las tasas de actividad de estos trabajadores que ahora no se está produciendo. En este análisis de las tasas de desempleo se sugiere que esta crisis también expulsa a los trabajadores de más edad del mercado laboral, pero que en este caso no se hace hacia la inactividad (o prejubilación), sino hacia el desempleo.

\section{Desempleo masculino, vuelco en el género del paro}

A primera vista llama la atención el vuelco que introduce la crisis en la feminización del desempleo. Durante el periodo expansivo, entre 2001 y 2008, las tasas de desempleo masculino son inferiores en casi medio punto a las del desempleo femenino, con un índice de masculinidad del desempleo situado en torno a 80 en la RMB y al 70 en la RMM. La crisis que se inicia en 2008 da un vuelco e invierte la situación, dando lugar a que las tasas de desempleo masculino superen en dos puntos

\footnotetext{
${ }^{6}$ Gómez y Miret, 2012.
} 
las del femenino en 2010 (figura 3). Esta masculinización remite, aunque más rápidamente en la $\mathrm{RMM}$ que en la $\mathrm{RMB}$.

Figura 3

Evolución de la proporción de paro registrado sobre población de 16 a 64 años según sexo

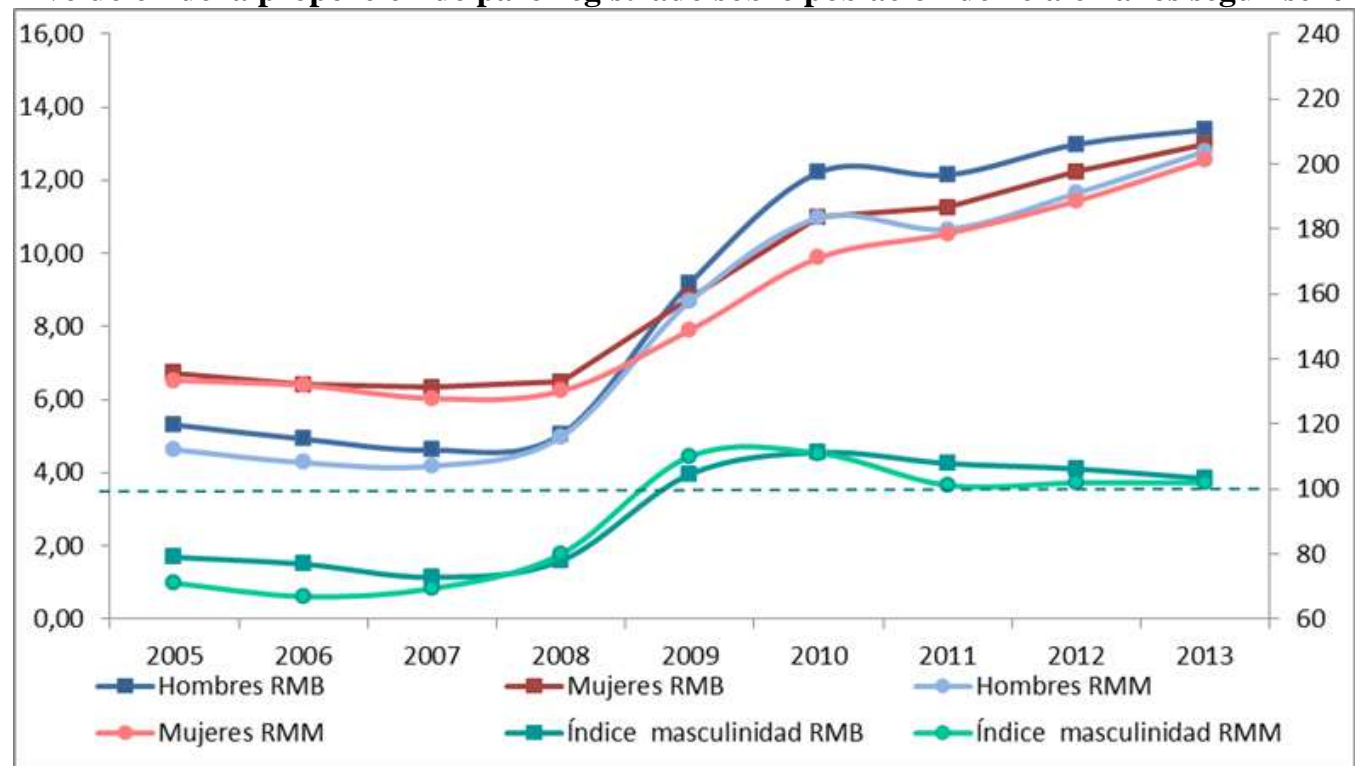

Fuente: Instituto de Estadística de Madrid, Observatori Metropolità del Treball, registros de desempleo. Los valores de 2013 se han estimado a partir de la los de 2012.

Los cambios en las tasas de desempleo según género tienen un componente marcadamente sectorial: el bajo porcentaje de mujeres empleadas en la construcción. Esta situación en la que el desempleo masculino sobrepasa al femenino, también se produce en la Región Metropolitana de Madrid, con un similar desarrollo de la construcción en los años previos a la crisis y con una gran importancia del sector servicios y del turismo en el empleo. Rocha y Aragón ${ }^{7}$ advierten de una diferencia en las tasas entre géneros: una parte del desempleo femenino no proviene de mujeres que han perdido su empleo, sino que responde al efecto del trabajador adicional. En este caso, algunas mujeres que buscan empleo porque sus parejas lo han perdido.

\section{Niveles de desempleo de la población extranjera}

Si el aumento del desempleo femenino daba pistas de la dualidad del mercado laboral, el cambio en las tasas de desempleo de la población extranjera a partir de 2008 la confirma. En este caso, la situación laboral relativamente más precaria de la población extranjera se combina con el efecto sectorial de la especialización laboral de los hombres extranjeros en la construcción. Gil, Bayona y Vono ${ }^{8}$ advierten de las mayores dificultades de integración derivadas de la movilidad provincial de los extranjeros en busca de oportunidades laborales. De manera similar Rinken ${ }^{9}$ señala los inconvenientes que el desempleo puede tener para la integración y asimilación de la población extranjera en nuestro país. Según datos de los registros de desempleo sobre el total de población, sus tasas pasan de situarse en torno al 4\% en 2008 a triplicarse en

\footnotetext{
${ }^{7}$ Rocha y Aragón, 2012.

${ }^{8}$ Gil, Bayona y Vono, 2011.

${ }^{9}$ Rinken, 2012.
} 
2012 (figura 4). Según los datos generales de la EPA que manejan Rocha y Aragón ${ }^{10}$, se estima que su desempleo alcanzaba al 38,7\% en 2012, 16 puntos más que las tasas que afectan a la población nacional. Las tendencias a la baja del último año (2013) deben tomarse con precaución, ya que al estimarse las poblaciones de referencia de 2013, la tasa no recogería ni las posibles reducciones recientes en el total de activos, ni posibles reducciones de población en 2013. Es decir, cuando toda la información de 2013 esté disponible, estas tasas de desempleo para la población de nacionalidad extrajera serán, previsiblemente, algo superiores.

Figura 4

Evolución de la proporción de paro registrado sobre población de 16 a 64 años de nacionalidad extranjera (eje izquierdo) y evolución del total de la población extranjera (derecho)

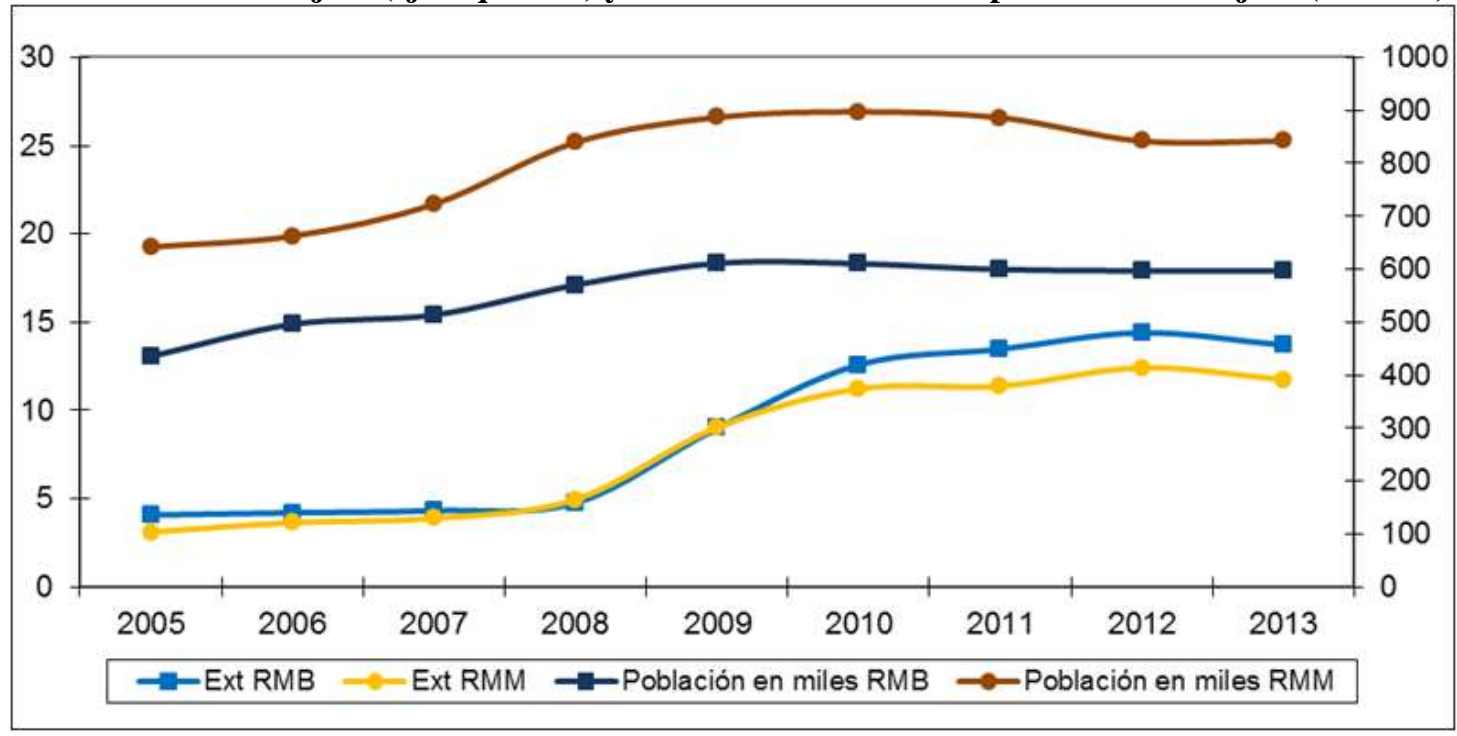

Fuente: Instituto de Estadística de Madrid, Observatori Metropolità del Treball, registros de desempleo; Padrón continuo de población, INE. Los valores de 2013 se han estimado a partir de los de 2012.

\section{Transformaciones en las tasas de desempleo registrado en función de las principales variables sectoriales y ocupacionales}

\section{Sectores de actividad, colapso de la construcción}

Las tasas brutas de la RMB (figura 5) dependen, en primer lugar, de la composición de la estructura sectorial del empleo en la $\mathrm{RMB}$, en la que predomina claramente el sector servicios.

Los dos rasgos más característicos que pueden apreciarse a partir del grafico de evolución de tasas de desempleo registrado (figura 5), son el salto cualitativo que produce el desempleo en la construcción en la primera crisis (2008-2010) y el continuo e intenso aumento del desempleo en el sector servicios a partir de 2010. Estos datos presentan el problema de no contar con una población de referencia agrupada por sectores. Como para la RMM se disponen de las cifras de registrados, se pueden

\footnotetext{
${ }^{10}$ Rocha y Aragón, 2012.
} 
ofrecer tasas que van más allá de la distribución porcentual del desempleo por sectores (figura 6).

Figura 5

Tasas de paro sobre población de 16 a 64 según el sector de actividad, RMB 2007-2013

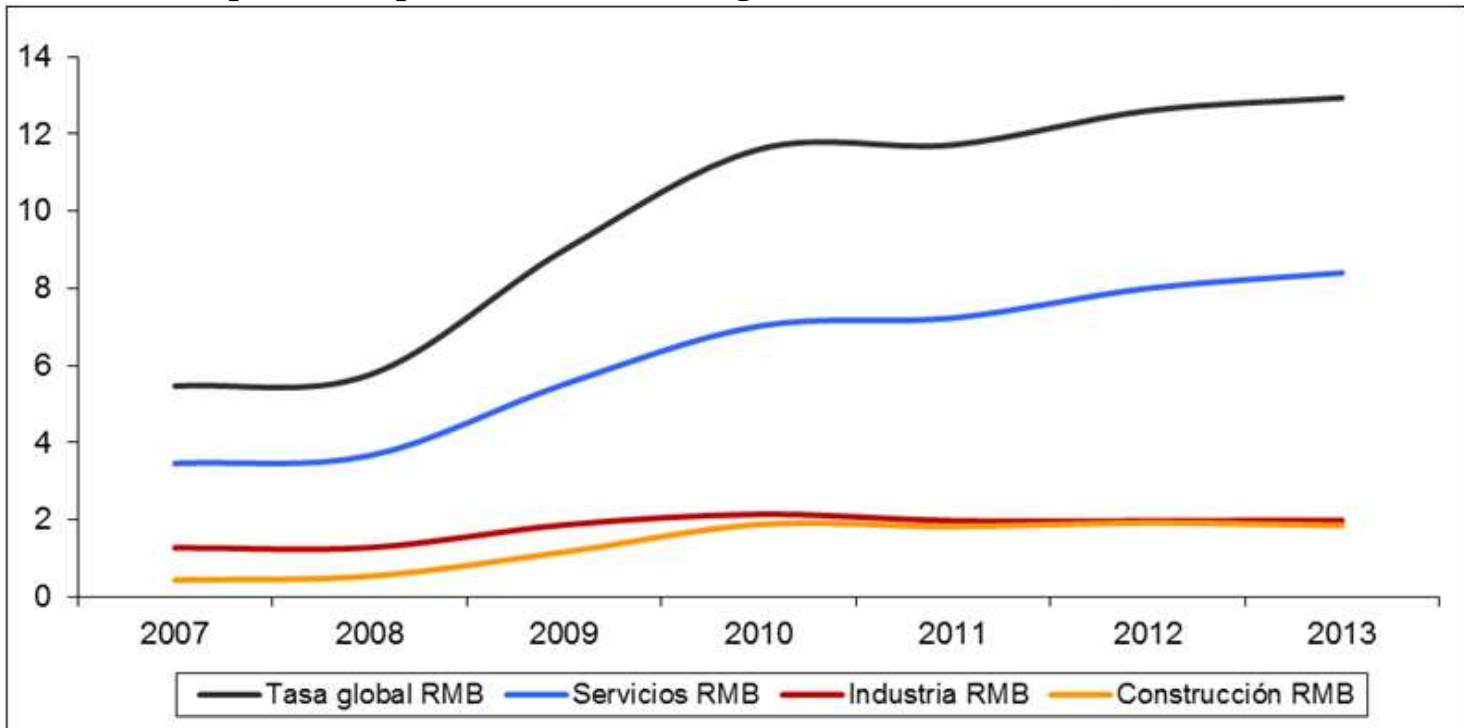

Fuente: Observatori Metropolità del Treball, registros de desempleo. Los valores de 2013 se han estimado a partir de los de 2012.

En la RMM destaca el fuerte crecimiento de las tasas de desempleados sobre ocupados por sectores, especialmente en el sector de la construcción. Si las tasas globales, en industria y servicios se llegan a triplicar pasando de valores próximos a $8 \%$ en 2007 , al $19,8 \%$ en los servicios y al $25,6 \%$ en la industria en 2013. El crecimiento de las tasas de desempleo sobre ocupados en la construcción es tan alto que, a partir de 2011, hay más trabajadores registrados como desempleados que como ocupados en este sector.

Figura 6

Tasas de paro registrado sobre ocupados según el sector de actividad, RMM 2007-2013

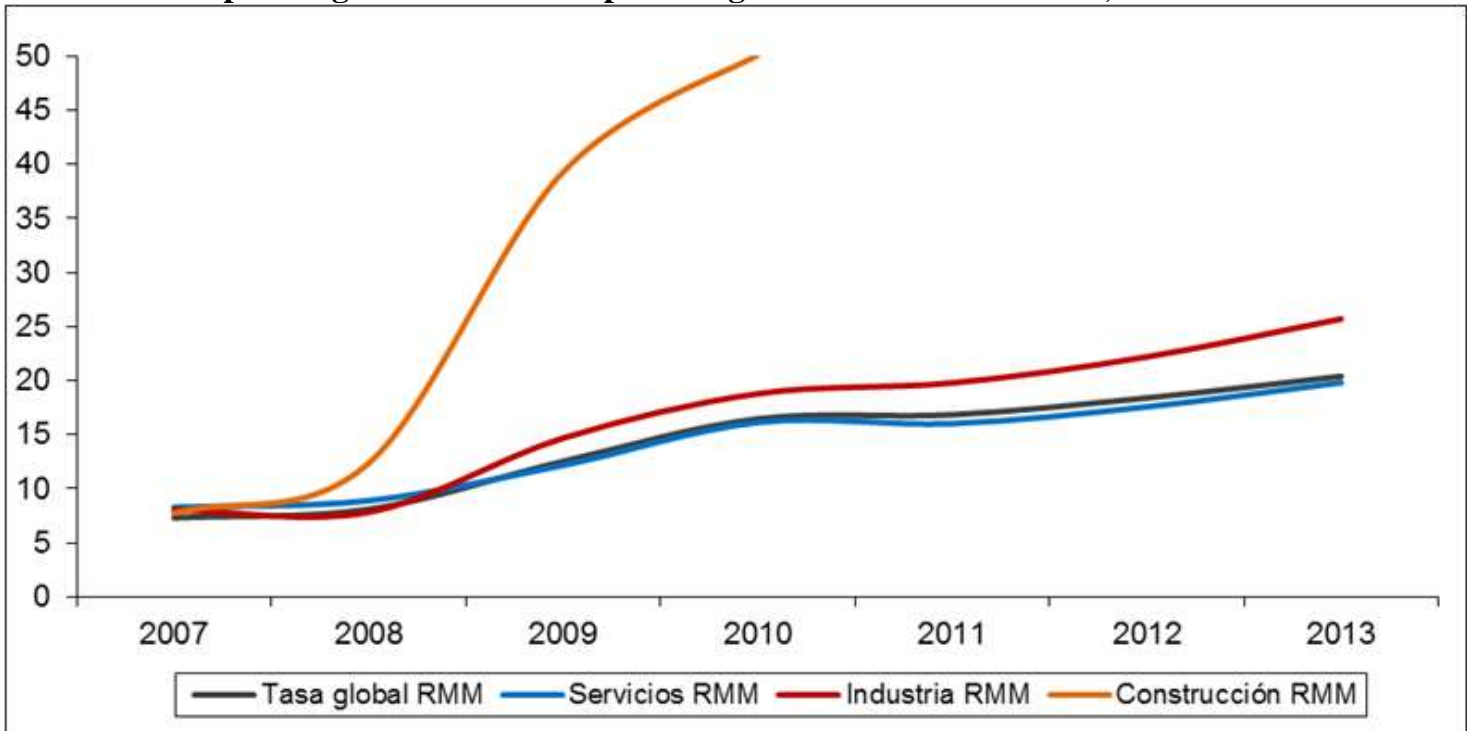

Fuente: Instituto de Estadística de Madrid, registros de desempleo y afiliados a la Seguridad Social. Los valores de 2013 se han estimado a partir de la población de 2012. 


\section{Las desigualdades del desempleo según categorías profesionales}

El principal rasgo de la evolución del desempleo por categorías ocupacionales en la RMB que ofrece la figura 7, es el aumento de la dispersión de las tasas a partir de la crisis de 2008. En general, si el periodo de desarrollo económico produjo cierta convergencia, la crisis ha acentuado las diferencias entre las tasas de desempleo de las categorías ocupacionales.

Figura 7

Evolución de las tasas de paro sobre población de 16 a 64 años por categorías ocupacionales, RMB

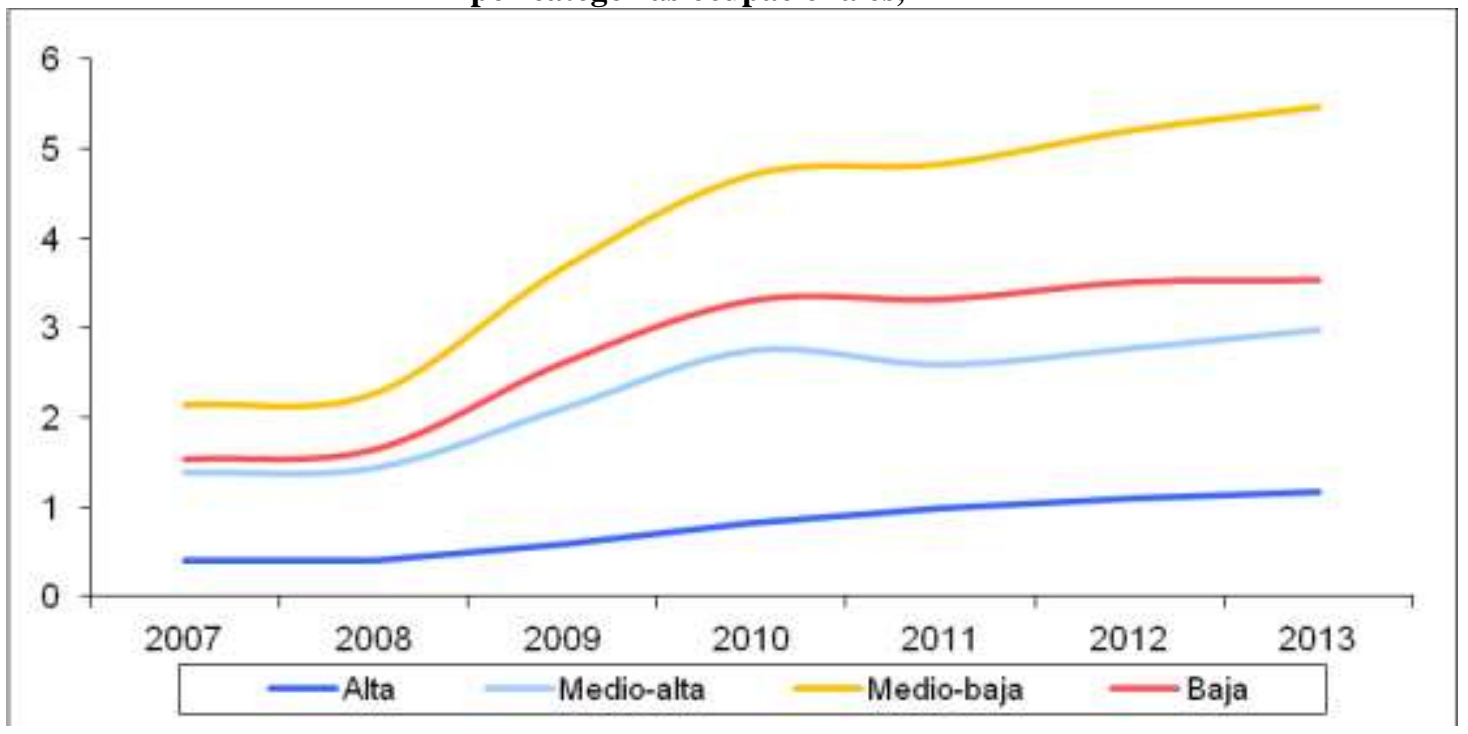

Fuente: Observatori Metropolità del Treball, registros de desempleo. Los valores de 2013 se han estimado a partir de la población de 2012.

Poco más puede decirse porque estas tasas de desempleo sobre la población potencialmente activa pueden estar indicando tanto cambios en el nivel de desempleo, como en la proporción de ocupados en cada una de las diferentes categorías. El cálculo de tasas sobre ocupados para la RMM es capaz de ofrecer una mayor precisión (ver figura 8), pero tampoco está exento de problemas. Para la RMM las tasas de desempleados registrados por categorías ocupacionales (CNO-grandes grupos) se calculan sobre los ocupados afiliados a la Seguridad Social por categorías (técnicamente por grupos de cotización).

Las dos clasificaciones no son coincidentes, ni están necesariamente estratificadas. Para aproximarlas a categorías ocupacionales, se deben generarse grandes agrupaciones que sean, a la vez, compatibles y estratificadas. En este caso, se han creado tres grandes categorías ocupacionales: alta, trabajadora y baja que permiten obtener cierta referencia de los niveles de desempleo según categorías ocupacionales en la RMM y su evolución. En el caso de la RMM, y previsiblemente ocurre de manera análoga en la RMB, el aumento del desempleo y la dispersión de tasas entre categorías que se producen a partir de 2008, afecta más a los grupos más bajos. Las tasas de desempleo de los trabajos de mayor categoría y cualificación se sitúan bajo la media, mientras que el grueso de los trabajadores sufre tasas por encima de la media. Esta mayor incidencia del desempleo es especialmente aguda en las categorías más bajas: entre los peones y las ocupaciones elementales se encuentran las mayores tasas de desempleo. 
Figura 8

Evolución de las tasas de paro registrado sobre ocupados por categorías ocupacionales, RMM

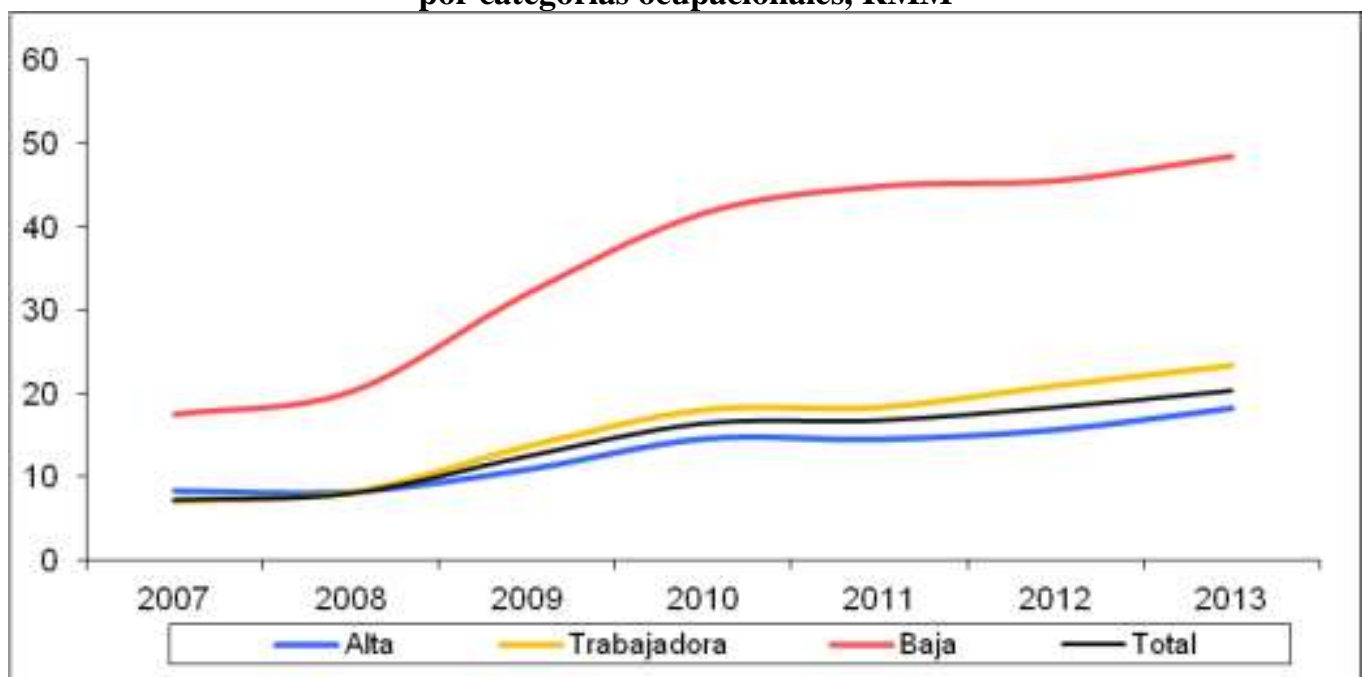

Fuente: Instituto de Estadística de Madrid, registros de desempleo y afiliados a la Seguridad Social. Los valores de 2013 se han estimado a partir de la población de 2012.

\section{Evolución de los agregados territoriales}

Una ventaja del uso de los registros decisiva para análisis territoriales es el acceso a datos con detalle municipal. Este nivel de desagregación permite tanto ofrecer características internas y cartografías de la distribución del desempleo en la RMB, como analizar las transformaciones recientes y el impacto de la crisis en función de las comarcas, del tamaño del municipio o de los grupos de municipios según su condición socioeconómica.

\section{Las comarcas}

En la serie de los últimos años, se producen tres grandes procesos a nivel comarcal: la polarización de las diferencias territoriales en los niveles de desempleo, la progresiva incorporación de comarcas de carácter suburbano a dinámicas metropolitanas y la reducción relativa de las comarcas centrales.

En la RMB (figura 9), estos cambios se aceleran a partir de 2008 e incluyen tanto la incorporación del Alt Penedès a dinámicas metropolitanas como un reposicionamiento de las tasas del Barcelonés en los niveles inferiores. El Alt Penedès parte de tasas muy bajas de desempleo y en 2012 alcanza una tasa de desempleados registrados sobre la población potencialmente activa equiparable a la del Baix Llobregat, del 13,4. El segundo gran cambio es el reposicionamiento de las tasas de desempleo del Barcelonès que se sitúa entre las comarcas con tasas relativamente más bajas de desempleo. En, este cambio, el municipio de Barcelona tiene un peso decisivo. La polarización de las diferencias territoriales se observa a partir del estallido de la crisis: las 3 comarcas en las que el desempleo evoluciona peor son las menos urbanizadas (Penedès, Vallès Oriental y Garraf). 
Figura 9

Evolución de la proporción del paro sobre población por comarcas seleccionadas, $\mathbf{R M B}$

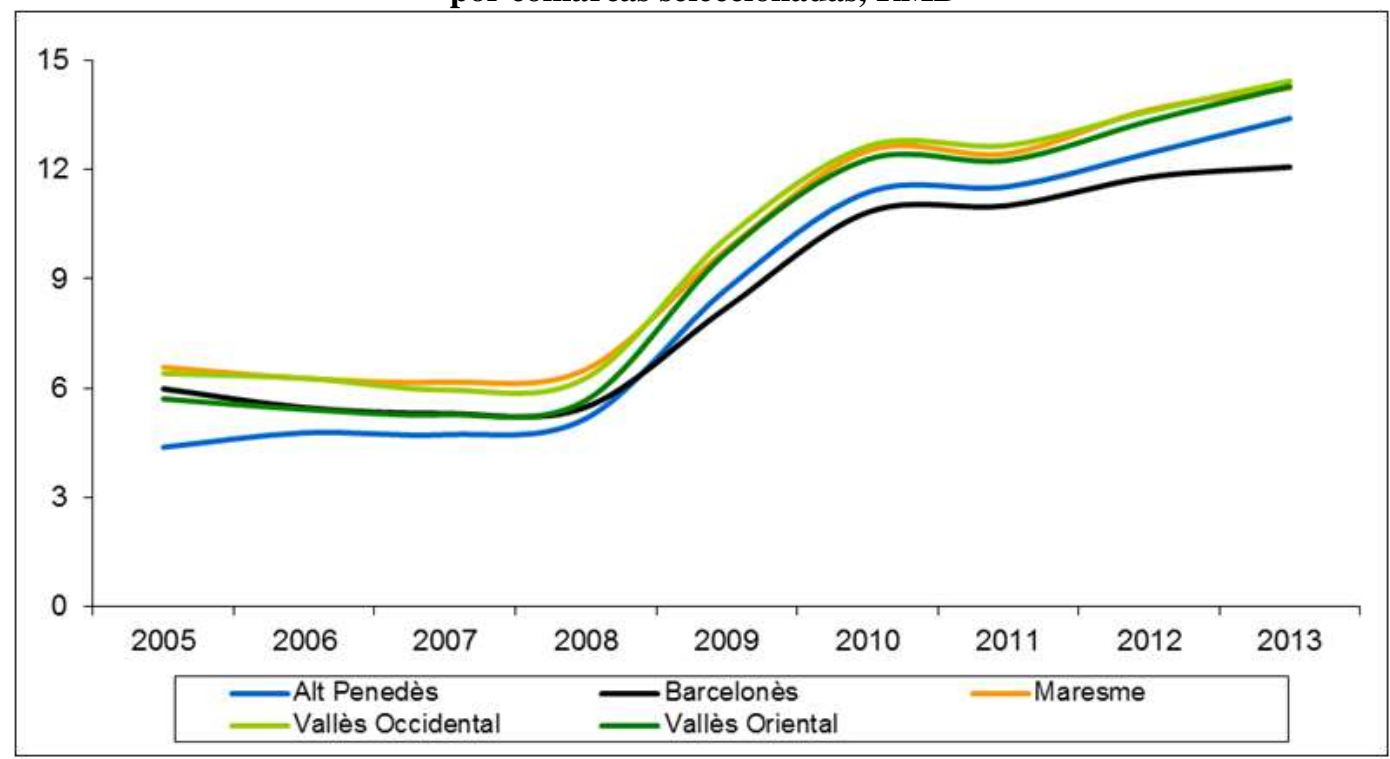

Fuente: Observatori Metropolità del Treball, registros de desempleo. Los valores de 2013 se han estimado a partir de la población de 2012.

En la RMM, la Sierra Norte es la comarca que, desde ubicaciones más periféricas, sufre mayores aumentos en sus tasas de desempleo, posiblemente relacionados con su creciente incorporación a dinámicas metropolitanas durante el periodo. Aunque en un proceso menos evidente que en el caso del Barcelonés, el municipio de Madrid reduce su nivel relativo de desempleo, alejándose de las tasas del este metropolitano y aproximándose a los niveles del norte y el oeste (figura 10). En la RMM, la evolución de las tasas de desempleo aumenta las polarización territorial en el empleo entre las comarcas del norte y el oeste por un lado y el las del sur y el este por otro, así como la diferencia entre comarcas centrales próximas a Madrid y las comarcas más periféricas.

\section{Figura 10}

Evolución de la proporción del paro registrado sobre población por comarcas, RMM

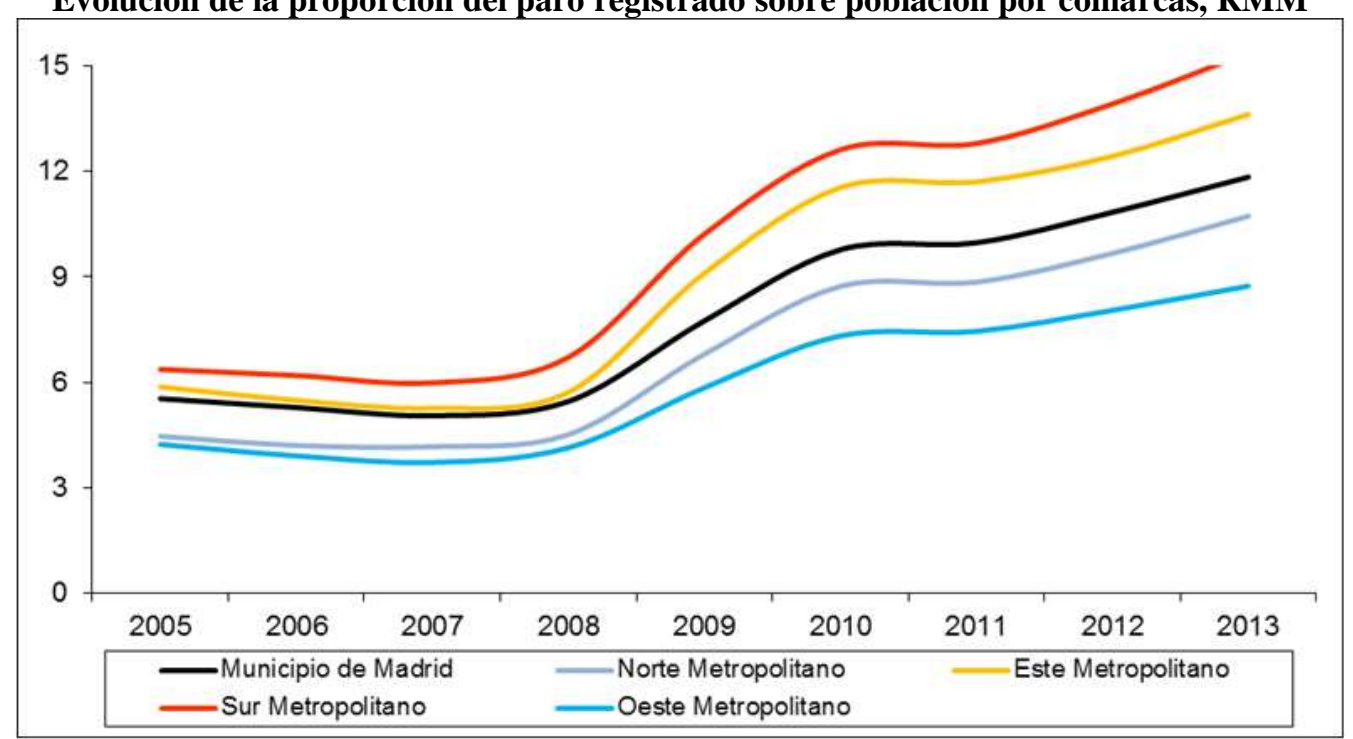

Fuente: Instituto de Estadística de Madrid, registros de desempleo. Los valores de 2013 se han estimado a partir de la población de 2012. 


\section{La dimensión del municipio}

Las tasas de desempleo en función del tamaño del municipio presentan una articulación y dinámicas similares en la RMB y la RMM: La ciudad central pasa de valores medios en 2005, a tener las menores tasas de desempleo de su respectiva región metropolitana (sobre el 10\% en 2013); las ciudades de más de 100.000 habitantes presentan tasas de paro relativamente altas, en torno al 16\% en 2013 (figuras 11 y 12). La principal divergencia es el papel de los municipios pequeños (5.000 y menos) y medios (entre 10.000 y 100.000). En la RMB los municipios pequeños tienen tasas bajas en 2005, pero en la RMM aunque los municipios pequeños parten también de tasas bajas (casi el 6\% en 2005), evolucionan mal durante la crisis y llegan en algunos casos a tener ratios superiores al 16\%. La situación se invierte con los municipios medios que en la RMB tienen tasas medio altas y en la RMM, bajas.

Figura 11

Tasas de paro registrado sobre población de 16 a 64, según tamaño de municipio, RMB

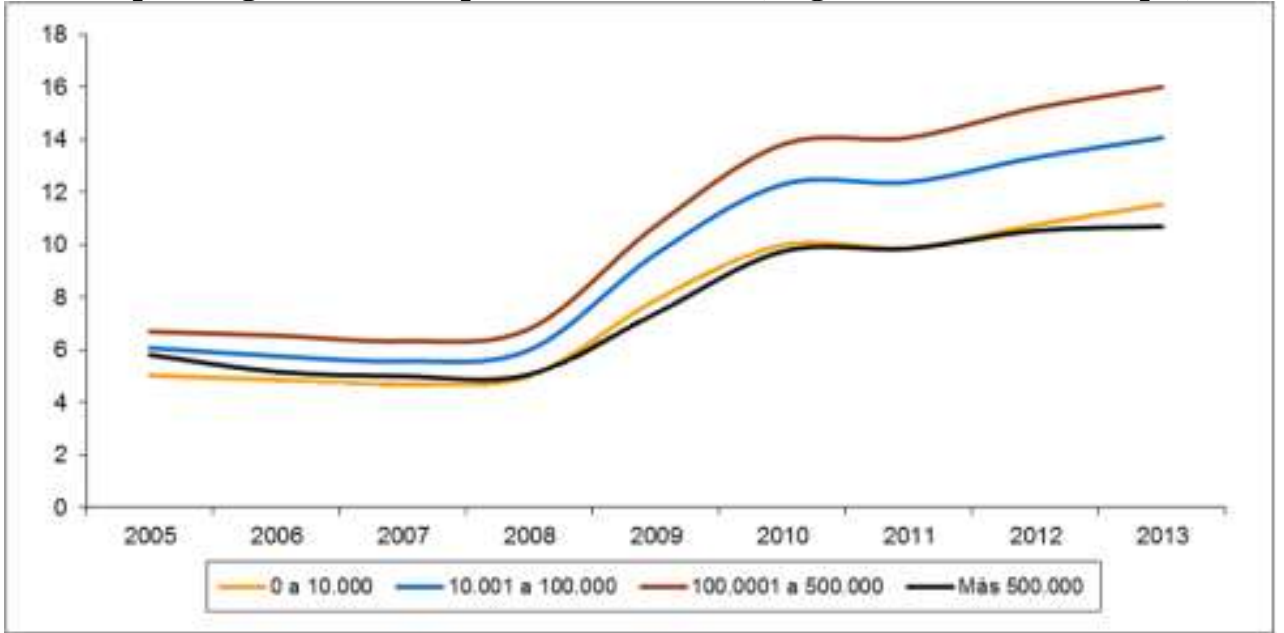

Fuente: Observatori Metropolità del Treball, registros de desempleo. Los valores de 2013 se han estimado a partir de la población de 2012.

Figura 12

Tasas de paro sobre población de 16 a 64 según dimensión de municipio, RMM

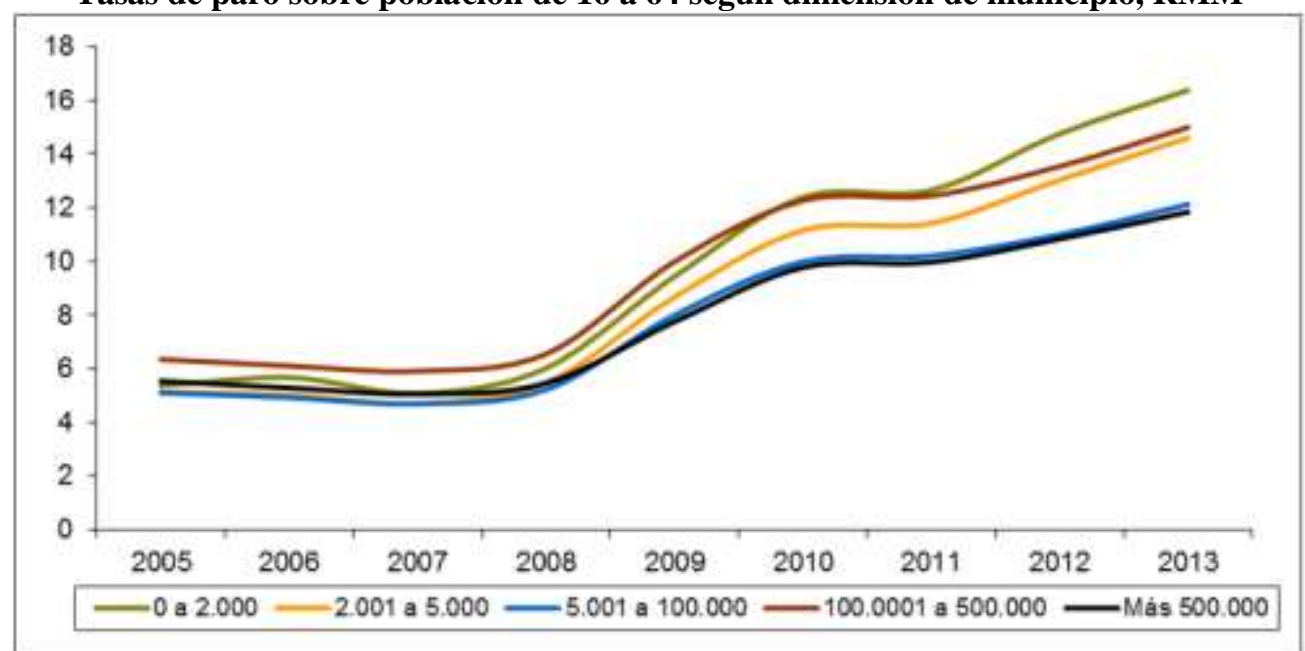

Fuente: Instituto de Estadística de Madrid, registros de desempleo. Los valores de 2013 se han estimado a partir de la población de 2012. 


\section{Condición socioeconómica del municipio}

Los análisis efectuados en función de las comarcas y del tamaño del municipio apuntan a una clave socioeconómica como factor de peso en los cambios de las tasas de desempleo. Esto explicaría tanto las diferencias entre ciudades centrales y el resto, como las bajas tasas de desempleo de las comarcas con concentración de municipios de clase alta (Oeste Metropolitano en la RMM, por ejemplo).

Figura 13

Evolución de las tasas de paro según categoría socioeconómica de los municipios, RMB

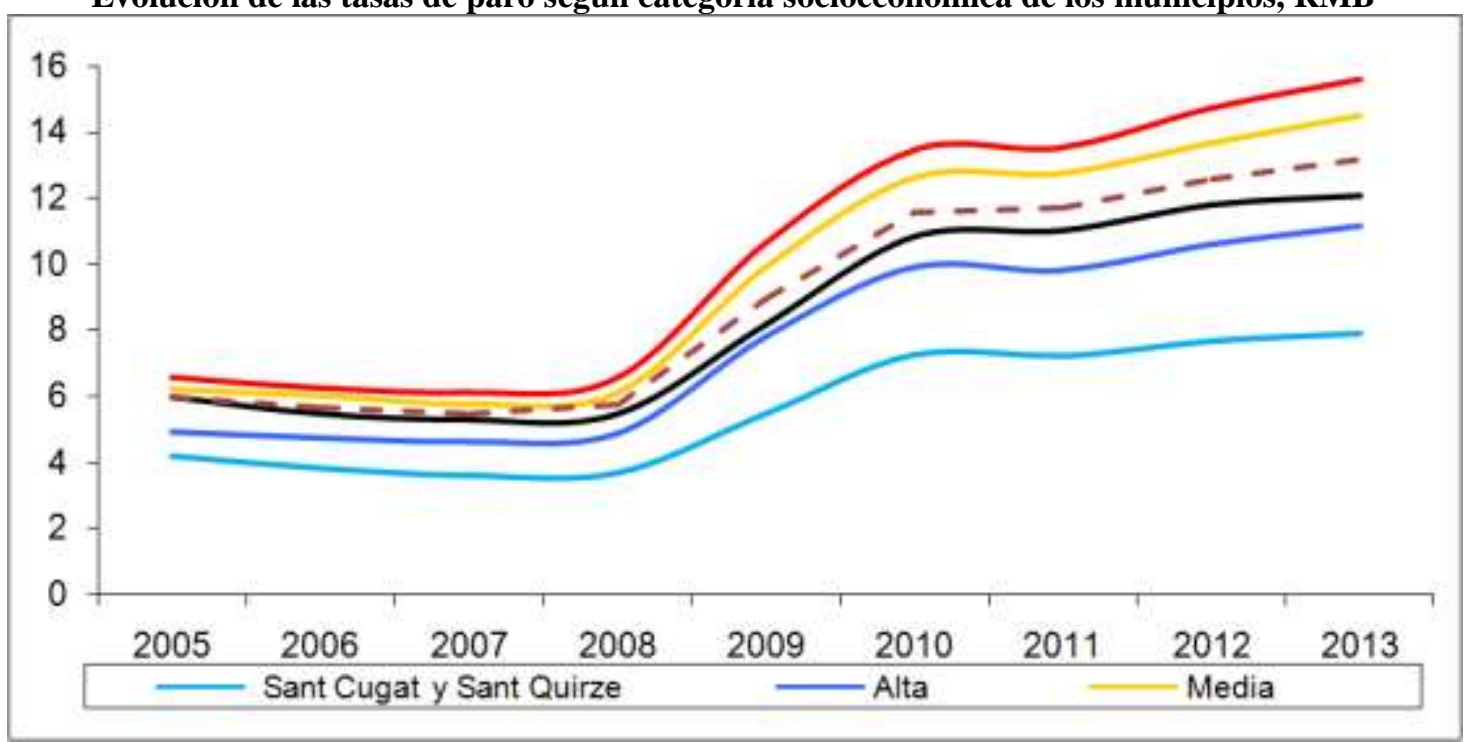

Fuente: Elaboración propia a partir de los registros de desempleo del Observatori Metropolità del Treball.

Para comprobarlo, se ha realizado una agrupación socioeconómica de los municipios que componen la RMB y la RMM a partir de datos del censo 2001 en función de la categoría ocupacional de la población residente ${ }^{11}$. Se han creado 4 grupos en función del porcentaje de ocupados en categorías alta, medio-alta, media, medio-baja y clase baja. Finalmente, se calcularon las tasas de paro registrado sobre población potencialmente activa para cada uno de estos agregados territoriales.

La relación entre la categoría socioeconómica y las tasas de desempleo es muy clara, tal como muestran las figuras 13 y 14, en las dos regiones metropolitanas existe una relación inversa entre categoría socioeconómica y nivel de desempleo. Hay que destacar la simetría de las estructura de clase y desempleo en ambas regiones.

Una parte de las diferencias entre las tasas de desempleo por categorías socioeconómicas en la RMB y la RMM se corresponden a diferencias en la escala de las unidades administrativas. El término municipal de Madrid es mayor que el de Barcelona. Así, las diferencias que se observan en las tasas entre ambos municipios se reducen al comparar Madrid con la comarca del Barcelonès. Ocurre un efecto de escala similar en los municipios de clase alta. En Madrid hay un mayor número de municipios de clase alta y gran homogeneidad en las inmediaciones de la ciudad central (generalmente, en el oeste metropolitano). En la RMB, sólo dos cumplen estas

\footnotetext{
${ }^{11}$ Siguiendo el análisis a nivel municipal de Rubiales, Bayona y Pujadas, 2012.
} 
características: Sant Cugat del Vallés y Sant Quirze del Vallés. Considerando estos dos municipios como el equivalente en la RMB a la clase alta de la RMM, observamos tasas de desempleo similares a las del conglomerado superior de la RMM.

Figura 14

Evolución de las tasas de paro según categoría socioeconómica de los municipios, RMM

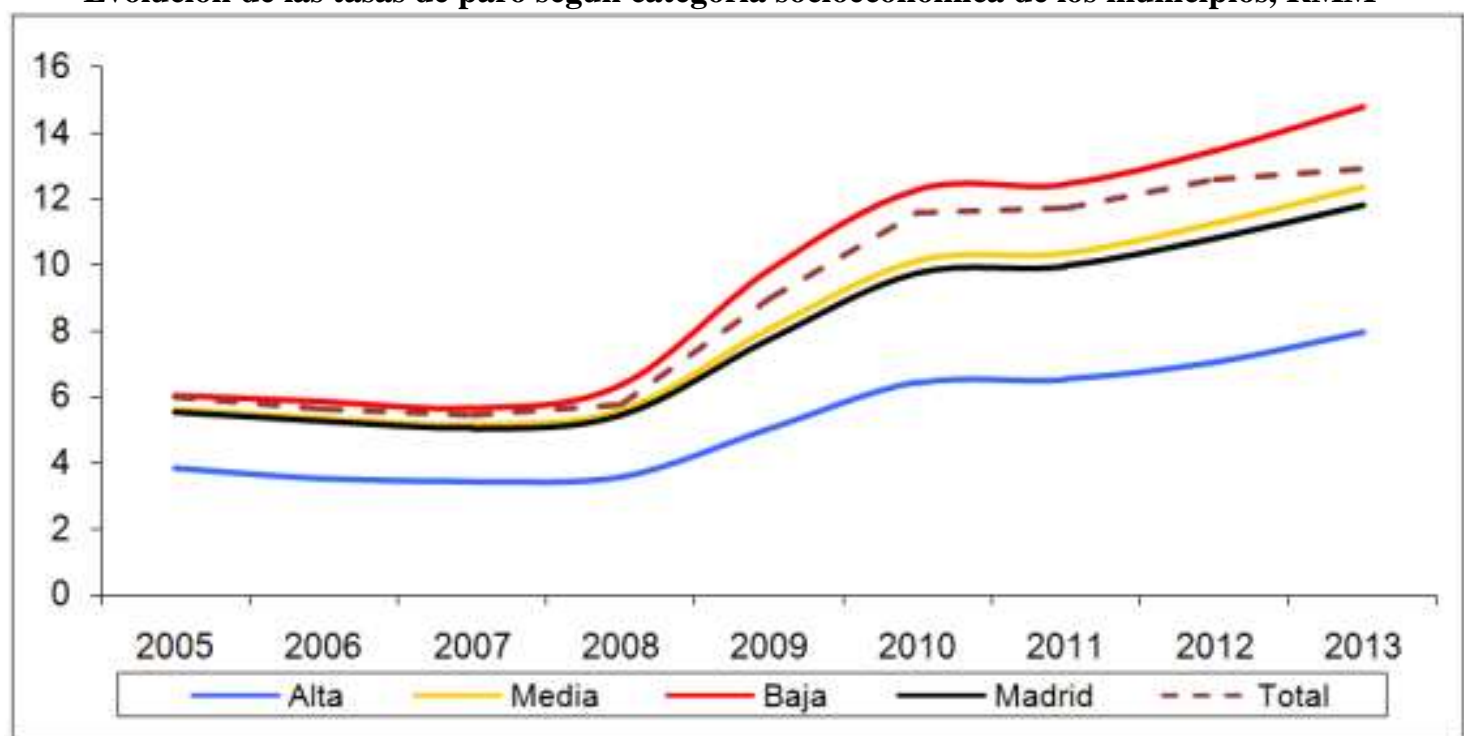

Fuente: Instituto de Estadística de Madrid, registros de desempleo. Los valores de 2013 se han estimado a partir de la población de 2012.

Tras estas correcciones, se mantienen diferencias sustantivas en el nivel de las tasas de desempleo y en su forma de evolución. Al superponer ambos gráficos, cada categoría socioeconómica de la RMB presenta, a partir de 2008, tasas sistemáticamente más altas que las de la RMM. Esta diferencia se debe a la mayor incidencia del desarrollo de la construcción y del colapso de este sector en la primera fase de la crisis. Esta incidencia diferencial de la crisis también se aprecia en la forma de las curvas. En la RMB la evolución de las tasas de desempleo, presenta subidas más pronunciadas de 2008 a 2010 y más moderadas de 2011 a 2013 que las que se producen en la RMM. La mayor homogeneidad socioeconómica de los municipios y escala de la segregación en la RMM son algunos de los factores que explicarían la mayor dispersión de las tasas de desempleo de la RMM en comparación con la RMB.

\section{Análisis multivariable}

Tras el seguimiento realizado en la evolución de las diferentes tasas, el análisis factorial permite, para cada periodo y región metropolitana, sintetizar las variables que hemos ido presentando hasta el momento. Lo que se hace en este análisis es agrupar las variables similares con información redundante en factores, o metavariables más generales. Por ejemplo: durante el periodo 2008-2010, si los aumentos de las tasas de desempleo de los inmigrantes, de los hombres y de los trabajadores de la construcción se producen de forma muy marcada en algunos municipios, el análisis factorial crearía una variable compuesta por estas tres, un factor principal, que se podría interpretar como desempleo relacionado con la construcción. Tras este primer paso que fija los grandes ejes internos del proceso de incremento de tasas de desempleo durante los años de crisis, los componentes principales, se inicia un análisis de conglomeración. 
La conglomeración es una forma de agrupar resultados reduciendo el error cometido al simplificar, pero no es un análisis en sí misma. Los grandes factores que hemos obtenido con el análisis factorial y funcionan como metavariables, nos sirven ahora para trazar perfiles que describan típicamente a un número de municipios similares. $\mathrm{Al}$ conglomerar, lo que se busca es agrupar las unidades que tienen un perfil similar. Por ejemplo: un grupo de municipios sería el de aquellos que solo tienen el factor de desempleo por construcción alto y otro bien diferente, el de aquellos que tienen altos tanto el factor de desempleo relacionado con la construcción como el factor relacionado con el desempleo en los servicios. En el primer caso, la crisis queda limitada a una crisis de empleo sectorial, y en el segundo tipo de municipios, la crisis supone un golpe al conjunto del mercado laboral. Así, conglomerando y representando los municipios que presentan resultados similares en el análisis factorial, podemos localizar la articulación territorial de los componentes de la crisis para cada región metropolitana y periodo.

\section{Burbuja inmobiliaria y crisis financiera, componentes del desempleo 2008-2010}

Los procesos que componen el deterioro general del mercado laboral durante la primera fase de la crisis en la RMB y RMM se pueden resumir en 4. Un componente o factor relacionado con el aumento del desempleo en servicios que explica, aproximadamente la mitad de la varianza muestral (cuanto más varianza muestral explica un factor, más importancia tiene dentro del proceso estudiado, en este caso, las transformaciones en el desempleo relacionadas con la crisis). Este componente incide más en los grupos sociales con mayores dificultades de integración laboral: extranjeros, mayores de 44 años, mujeres y categorías ocupacionales bajas. El esquema es muy similar tanto en la RMM como en la RMB, aunque en esta última el componente de servicios, se subdivide en dos: el desempleo que afecta a los más mayores y el que afecta a extranjeros y mujeres. En la RMM, con ligeras variaciones, estos dos componentes se encuentran fusionados en un gran componente de servicios (cuadros 2 y 3 ).

Los otros tres factores explican el $20 \%$ y el $28 \%$ de la variabilidad muestral en la RMM y la RMB, respectivamente. Tienen un papel secundario y dan cuenta del desempleo sectorial en la construcción, en las zonas suburbanas y en la industria (previsiblemente, la industria de suministros a la construcción). Si el factor de construcción es el que más contribuye a la fuerte masculinización del desempleo producida durante el periodo, el industrial y el suburbano tienen un sesgo que perjudica las edades más avanzadas.

$\mathrm{Al}$ conglomerar estos factores se generan varias agrupaciones territoriales (figuras $15 \mathrm{a}$ 18): un primer grupo de municipios (C1) en los que se produce una fuerte destrucción del empleo, en la RMM está relacionado con un aumento generalizado en todos los sectores y en la RMB, con los puestos ocupados por trabajadores inmigrantes que trabajan en la industria de suministros para la construcción, en la construcción (factor 5) y también en los servicios (factor 1). Este conglomerado se representa en las figuras 19 y 20 en tonos anaranjados. 
Cuadro 2

Componentes principales del cambio en las tasas de desempleo 2008-2010, RMB

\begin{tabular}{|l|r|r|r|r|r|}
\hline & \multicolumn{5}{|c|}{ Componentes variación tasas de paro 2008-2010 } \\
\cline { 2 - 6 } & Extranjeros & $\begin{array}{c}\text { Mayores 44 } \\
\text { años }\end{array}$ & Construcción & Suburbano & Industria \\
\hline Agricultura & 0,150 & $-0,123$ & 0,141 & 0,874 & $-0,038$ \\
Industria & 0,181 & 0,342 & $-0,105$ & 0,122 & 0,840 \\
Construcción & 0,320 & $-0,110$ & 0,590 & $-0,047$ & 0,448 \\
Servicios & 0,539 & 0,524 & 0,012 & $-0,014$ & $-0,593$ \\
Alta & 0,030 & 0,375 & 0,046 & 0,729 & 0,170 \\
Media baja & 0,341 & 0,765 & 0,172 & $-0,132$ & $-0,003$ \\
Baja & 0,729 & 0,099 & $-0,096$ & 0,173 & 0,059 \\
Nac. extrnajera & 0,780 & $-0,033$ & 0,142 & 0,054 & 0,229 \\
Mujeres & 0,692 & 0,492 & $-0,445$ & $-0,026$ & 0,178 \\
Hombres & 0,412 & 0,512 & 0,702 & 0,179 & $-0,038$ \\
Índice Masculinidad & $-0,100$ & 0,087 & 0,929 & 0,154 & $-0,149$ \\
Menores de 30 años & 0,769 & 0,185 & 0,206 & $-0,109$ & $-0,019$ \\
De 30 a 44 años & 0,736 & 0,273 & 0,084 & 0,146 & $-0,106$ \\
De 45 a 64 años & 0,019 & 0,864 & $-0,018$ & 0,221 & 0,170 \\
Total & 0,676 & 0,688 & 0,144 & 0,147 & 0,051 \\
\hline
\end{tabular}

Fuente: Elaboración propia a partir de los registros de desempleo del Observatori Metropolità del Treball.

Cuadro 3

Componentes principales del cambio en las tasas de desempleo 2008-2010, RMM

\begin{tabular}{|l|r|r|r|r|}
\hline & \multicolumn{3}{|c|}{ Componentes variacion tasas de paro 2008 - 2010 RMM } \\
\cline { 2 - 5 } & \multicolumn{1}{|c|}{ Servicios } & Construccion & \multicolumn{1}{c|}{ Suburbano } & Industrial Mayor \\
\hline Industria & 0,094 & $-0,028$ & $-0,036$ & 0,964 \\
Construccion & $-0,012$ & 0,894 & 0,294 & $-0,030$ \\
Servicios & 0,953 & 0,191 & 0,031 & $-0,084$ \\
\hline Alta & 0,244 & 0,083 & 0,882 & $-0,008$ \\
Trabajadora & 0,887 & 0,253 & 0,083 & 0,172 \\
Baja & 0,355 & 0,580 & $-0,480$ & 0,156 \\
Extranjeros & 0,271 & 0,807 & $-0,078$ & $-0,106$ \\
Hombres & 0,266 & 0,875 & $-0,007$ & 0,089 \\
Mujeres & 0,966 & $-0,003$ & 0,087 & 0,001 \\
De 25 a 44 & 0,733 & 0,579 & $-0,092$ & 0,013 \\
\hline Mas de 44 & 0,669 & 0,214 & 0,335 & 0,308 \\
Total & 0,801 & 0,550 & 0,057 & 0,175 \\
\hline
\end{tabular}

Fuente: Instituto de Estadística de Madrid, registros de desempleo. Los valores de 2013 se han estimado a partir de la población de 2012.

Después viene un segundo grupo de municipios (C2) en el que se acentúa el aumento del desempleo en servicios en la RMB y el industrial en la RMM. En este conglomerado, tienen un mayor peso comparativo los trabajadores mayores de 44 años $y$, en la RMB, la población extranjera. Estos municipios se representan en la figura en tonos rojos. Un tercer grupo (C3) presenta valores bajos en los factores que componen el aumento del desempleo. Éste es el grupo que mejor resiste el impacto de la crisis, que tiene mayor población, un buen acceso a la ciudad central y o una composición poblacional de categoría relativamente más alta (en tonos azules en el mapa). 
Figura 15

Valores medios de los factoriales en cada uno de los conglomerados, 2008-2010, RMB

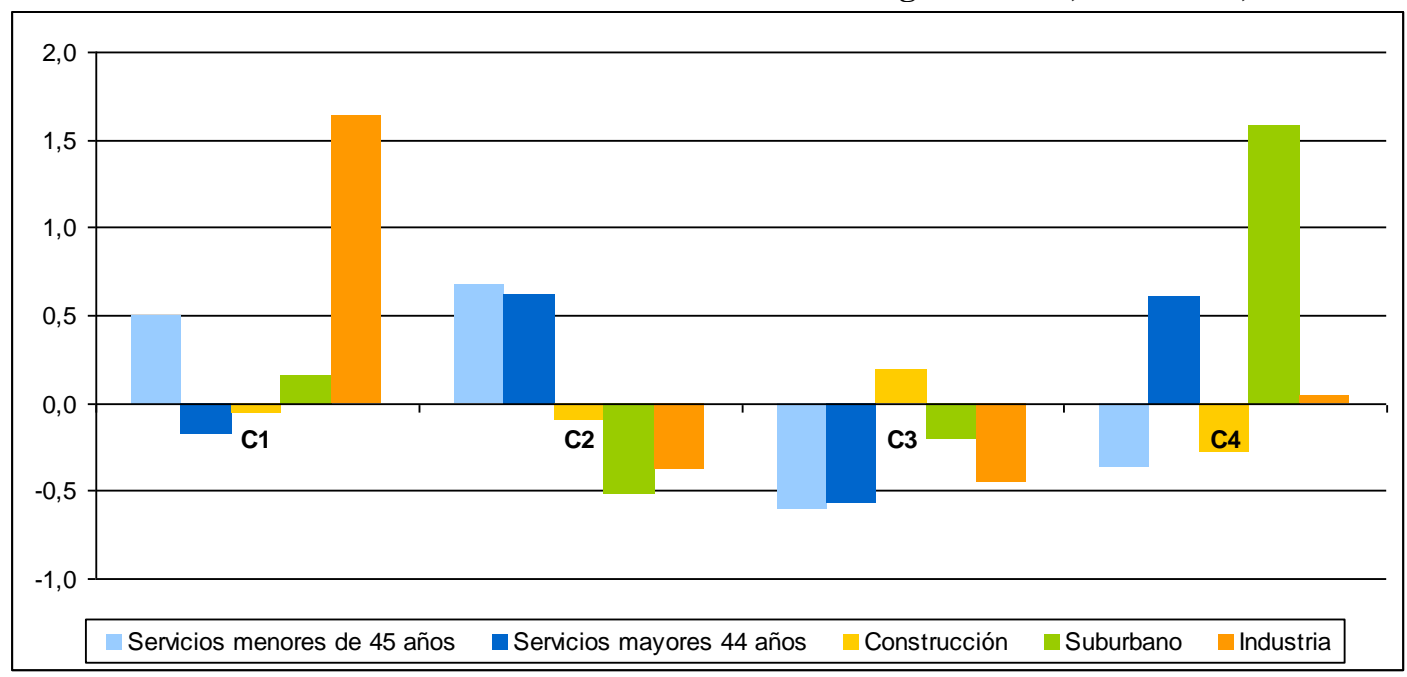

Fuente: Elaboración propia a partir de los registros de desempleo del Observatori Metropolità del

Treball.

Figura 16

Valores medios de los factoriales en cada uno de los conglomerados, 2008-2010, RMM

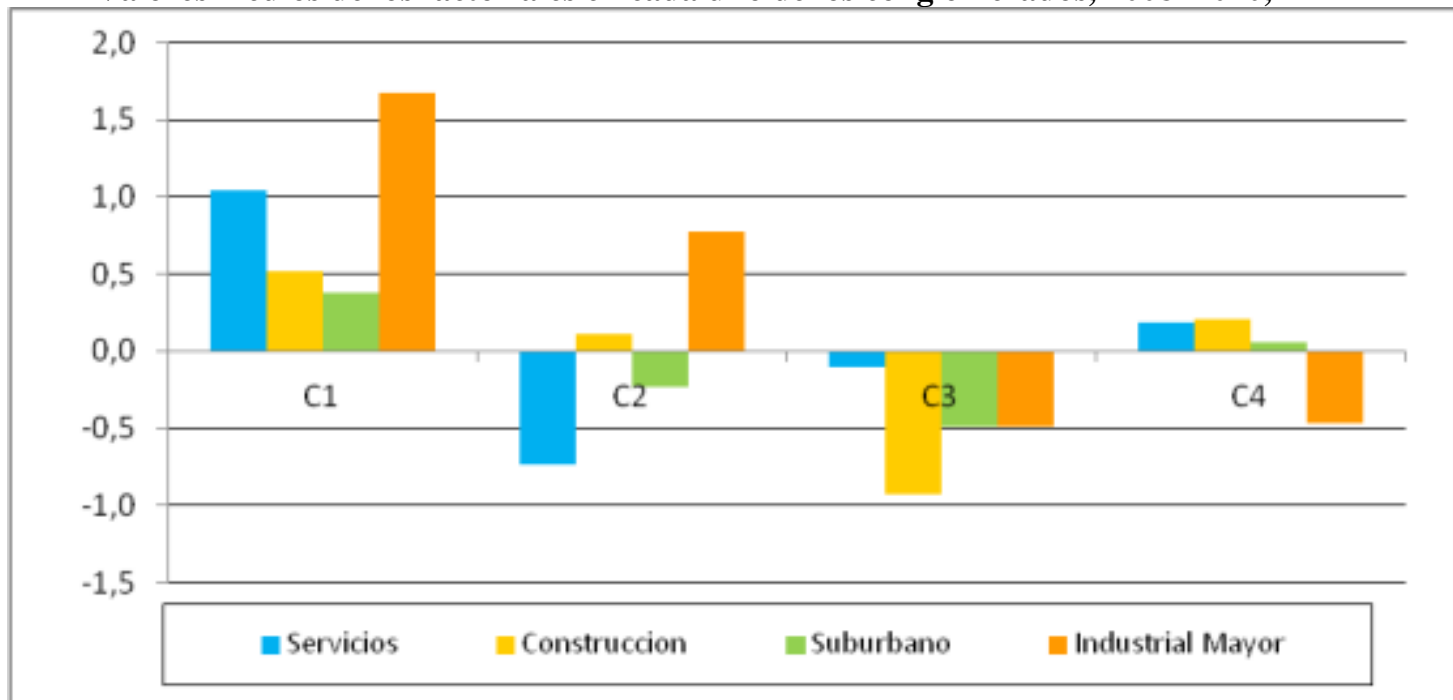

Fuente: Instituto de Estadística de Madrid, registros de desempleo. Los valores de 2013 se han estimado a partir de la población de 2012.

Finalmente, el último grupo $(\mathrm{C} 4)$ presenta perfiles muy dispares según la región metropolitana. En la RMB, combina el aumento de desempleo agrícola, de los trabajadores mayores del sector servicios y de los trabajadores de clases altas residentes en urbanizaciones de la zona. En la RMM viene a ser un grupo de municipios en los que tienen una mayor incidencia relativa el desempleo de servicios, con todo lo que conlleva, y el desempleo en construcción. 
Figura 17

Conglomerados de la evolución del desempleo en la RMB, 2008-2010

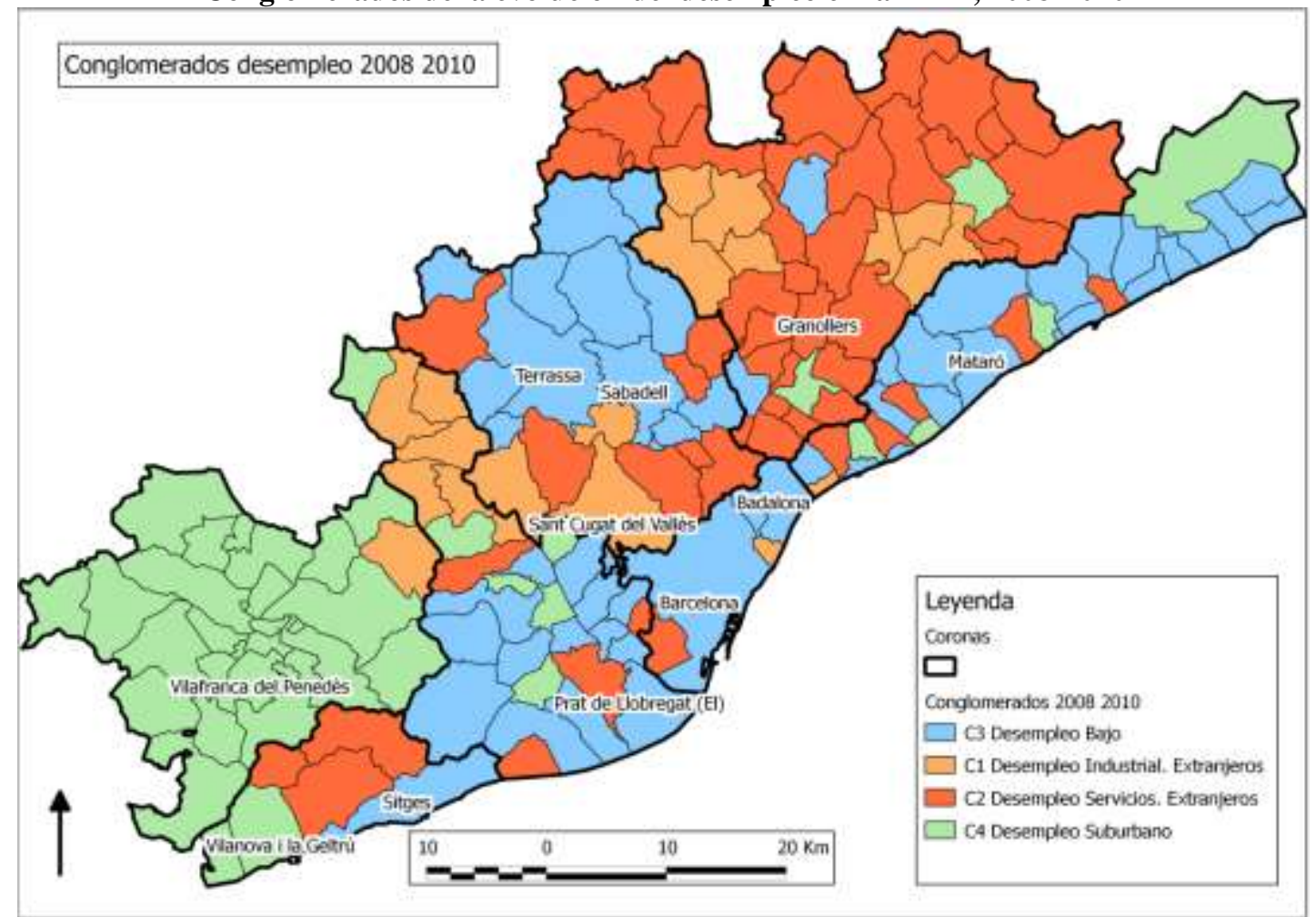

Fuente: Elaboración propia a partir de los registros de desempleo del Observatori Metropolità del Treball.

Figura 18

Conglomerados de la evolución del desempleo en la RMM, 2008-2010

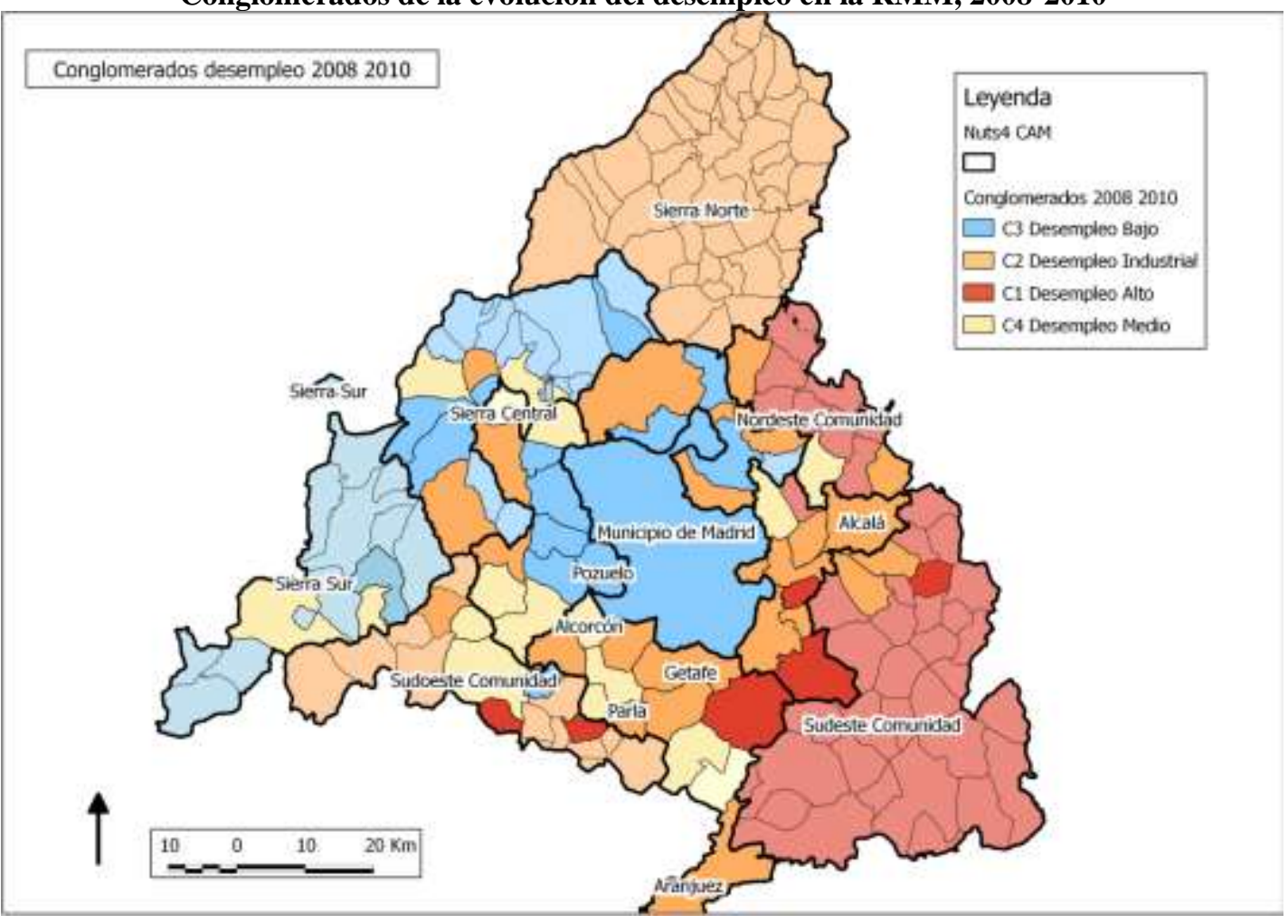

Fuente: Instituto de Estadística de Madrid, registros de desempleo. Los valores de 2013 se han estimado a partir de la población de 2012. 


\section{Desempleo y austeridad, componentes del desempleo 2011-2013}

El aumento de las tasas de desempleo durante este periodo no es tan intenso como en el periodo anterior, en parte porque ya se han descontado las burbujas, en parte porque el desempleo se encuentra en niveles muy altos. En este periodo, los procesos comienzan a presentar una mayor variedad interna en la RMB y divergencias entre Madrid y Barcelona. Mientras que en el análisis factorial de la RMB aparecen componentes específicos según categoría profesional, características sociodemográficas y localización (cuadro 4), en la RMM estos rasgos se solapan en los dos primeros componentes, sobretodo en el primero, relativo al desempleo del sector servicios (cuadro 5).

Las divergencias entre la RMB y la RMM pueden resumirse en que si en la RMB el desempleo relacionado con la industria y la construcción es residual, en la RMM siguen teniendo una presencia importante, posiblemente porque la reducción de la actividad en la construcción no fue tan intensa durante el primer periodo en la RMM, pero se está extendiendo durante más tiempo. En la RMB se sigue manteniendo aumentos del desempleo para la población extranjera (factor 3) y los trabajadores mayores (factores 2 y 6), pero a ellos se les añade un importante desempleo femenino (factor 1 con $23 \%$ de varianza explicada) y de clases trabajadoras (factor 4 ).

Cuadro 4

Componentes principales del cambio en las tasas de desempleo, 2011-2013, RMB

\begin{tabular}{|c|c|c|c|c|c|c|}
\hline & \multicolumn{6}{|c|}{ Componentes variación tasas de paro 2011-2013 } \\
\hline & Femenino & $\begin{array}{c}\text { Masculino y } \\
\text { mayor }\end{array}$ & $\begin{array}{c}\text { Extranjeros y } \\
\text { suburbano }\end{array}$ & Media baja & $\begin{array}{c}\text { Menores de } \\
30 \text { años }\end{array}$ & $\begin{array}{c}\text { Mayores de } \\
44 \text { años }\end{array}$ \\
\hline Agricultura & $-0,106$ & 0,061 & 0,596 & $-0,296$ & 0,034 & 0,327 \\
\hline Industria & $-0,082$ & 0,707 & 0,043 & $-0,142$ & $-0,054$ & $-0,156$ \\
\hline Construcción & $-0,155$ & 0,376 & 0,687 & 0,038 & 0,005 & 0,025 \\
\hline Servicios & 0,264 & 0,315 & 0,307 & 0,425 & 0,489 & 0,187 \\
\hline Total & 0,277 & 0,686 & 0,297 & 0,289 & 0,350 & $-0,026$ \\
\hline Alta & 0,323 & 0,007 & $-0,335$ & $-0,099$ & 0,392 & 0,347 \\
\hline Media alta & 0,031 & $-0,069$ & 0,102 & $-0,015$ & 0,031 & 0,891 \\
\hline Media baja & 0,096 & 0,145 & $-0,041$ & 0,843 & 0,085 & 0,000 \\
\hline Baja & 0,255 & 0,123 & $-0,066$ & $-0,842$ & 0,197 & 0,045 \\
\hline Nac. extranjera & 0,188 & $-0,081$ & 0,744 & 0,210 & 0,190 & $-0,113$ \\
\hline Mujeres & 0,784 & 0,343 & 0,246 & 0,224 & 0,224 & 0,124 \\
\hline Hombres & $-0,404$ & 0,736 & 0,272 & 0,224 & 0,294 & 0,118 \\
\hline Índice Masculinidad & $-0,902$ & 0,340 & 0,044 & 0,036 & 0,091 & $-0,011$ \\
\hline Menores de 30 años & $-0,135$ & 0,011 & 0,147 & $-0,079$ & 0,798 & $-0,054$ \\
\hline De 30 a 44 años & 0,313 & 0,352 & 0,427 & $-0,041$ & 0,135 & 0,066 \\
\hline De 45 a 64 años & 0,122 & 0,592 & $-0,061$ & 0,103 & $-0,269$ & 0,474 \\
\hline
\end{tabular}

Fuente: Elaboración propia a partir de los registros de desempleo del Observatori Metropolità del

Treball.

En la RMM hay un gran componente que explica, aproximadamente, la mitad de la varianza (cuadro 5). En él, se solapan varios procesos de aumento del desempleo en servicios: el de refeminización del desempleo, de sustitución de los trabajadores de más edad y mejores condiciones laborales por nuevos contratos y, en general, una mayor incidencia del desempleo en los colectivos más vulnerables, como la población extranjera y las categorías profesionales bajas. Una marcada diferencia con la RMB es el contraste de la industria en este periodo. Si en la RMB los municipios de especialización industrial se suman, en esta segunda fase de la crisis, al grupo de municipios más resistentes al desempleo, en la RMM el desempleo industrial en 2011 
sigue siendo un componente importante del aumento del desempleo (30\% de varianza total explicada).

Cuadro 5

Componentes principales del cambio en las tasas de desempleo 2011-2013, RMM

\begin{tabular}{|l|r|r|r|}
\hline \multirow{2}{*}{} & \multicolumn{2}{|c|}{ Componentes variacion tasas de paro 2011 - 2013 } \\
\cline { 2 - 4 } & Servicios & Industrial & Construccion \\
\hline Industria & $-0,210$ & 0,750 & $-0,271$ \\
\hline Construccion & 0,075 & 0,028 & 0,950 \\
\hline Servicios & 0,765 & 0,536 & 0,062 \\
\hline Alta & 0,723 & 0,015 & 0,100 \\
Trabajadora & 0,080 & 0,936 & 0,098 \\
Baja & 0,752 & 0,100 & 0,014 \\
Extranjeros & 0,839 & 0,059 & $-0,050$ \\
Hombres & 0,346 & 0,858 & 0,018 \\
Mujeres & 0,765 & 0,178 & 0,196 \\
De 25 a 44 & 0,486 & 0,784 & 0,224 \\
Mas de 44 & 0,848 & 0,216 & 0,047 \\
Total & 0,648 & 0,723 & 0,172 \\
\hline
\end{tabular}

Fuente: Instituto de Estadística de Madrid, registros de desempleo. Los valores de 2013 se han estimado a partir de la población de 2012.

De cara a su articulación territorial (figuras 19 a 22), los factores se agrupan en 6 conglomerados en la RMB (de los que se descartan 2 formados por atípicos), y en 8 en la RMM (5 atípicos). Los atípicos con grupos formados por uno o dos elementos con valores tan extremos que se diferencian de todos los demás. El tratamiento que se hace de ellos es no excluirlos del análisis. Como de cara a la representación pueden dificultar la comprensión general, se ha optado por asimilarlos cromáticamente al conglomerado con el que tengan un perfil más similar. Por ejemplo, los atípicos de valores extremadamente altos de desempleo en la construcción se asimilan al conglomerado en el que se aglutinan los municipios con un perfil marcado de desempleo alto en la construcción.

En esta segunda fase de crisis, existe un grupo de municipios común que resiste el desempleo mejor que los demás (C1). Se representa en tonos azules (figura 19 y 20). En este grupo de menor aumento del desempleo, continúan Madrid y los municipios del oeste metropolitano en la RMM; mientras que en la RMB, resisten mejor Barcelona, los municipios del noroeste de Barcelona y Sitges. Además se añaden al grupo de "resistentes" en la RMB los municipios del norte de Barcelona, los de especialización industrial y los de clase alta del Maresme.

Un cambio similar ocurre en la RMB: se incorporan al conglomerado de menos desempleo los municipios de mayor población y proximidad a Madrid y el eje más industrial de Alcalá. La principal diferencia en la evolución de este conglomerado en las dos regiones, es que los municipios de categoría socioeconómica baja más próximos a Madrid resisten mejor el desempleo que sus equivalentes en Barcelona. En la RMM, la evolución del desempleo durante el segundo periodo tiende a escalonarse más en función de la población y la distancia a la capital, mientras que en este periodo, la caracterización sociodemográfica de los municipios juega un papel importante en la RMB. En este sentido, el proceso de degradación del empleo en Madrid y Barcelona 
tendría una incidencia territorial similar, sólo que en la RMB comenzó antes y en la RMM los componentes sectoriales aun predominan frente a los sociales.

Figura 19

Valores medios de los factoriales en cada uno de los conglomerados, 2011-2013

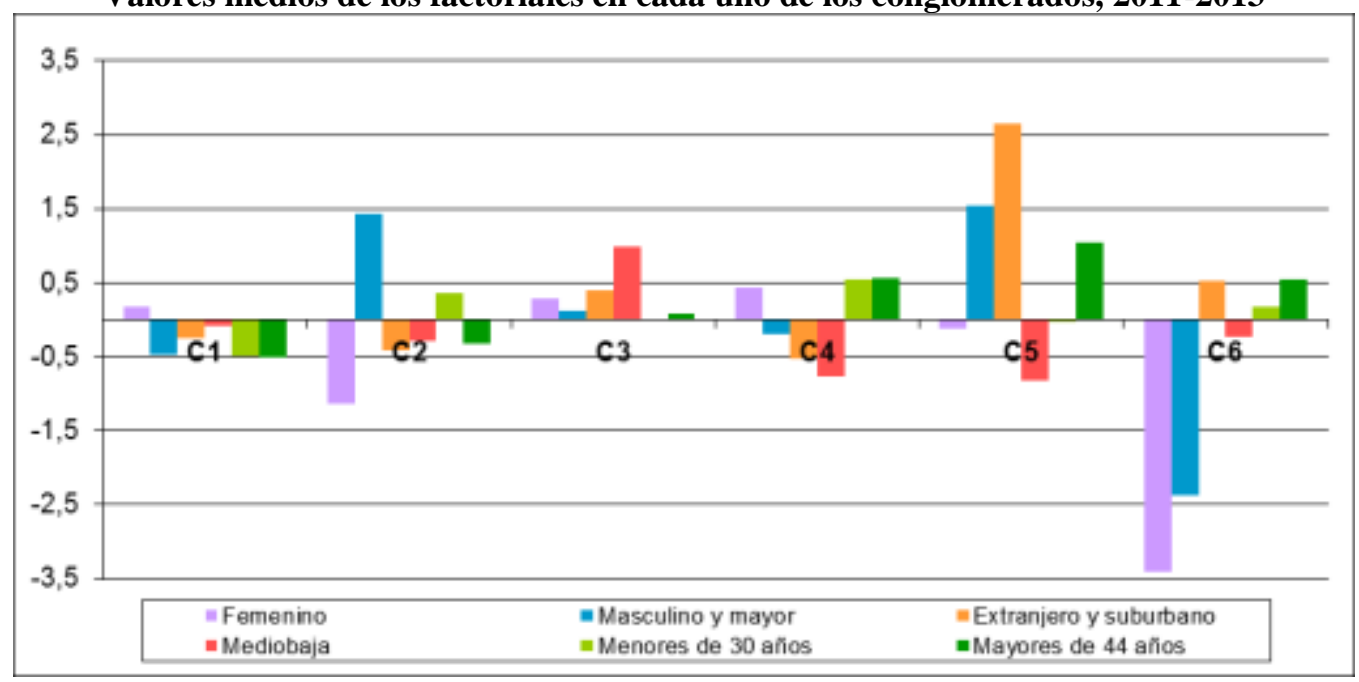

Fuente: Elaboración propia a partir de los registros de desempleo del Observatori Metropolità del Treball.

Figura 20

Valores medios de los factoriales en cada uno de los conglomerados, 2011-2013

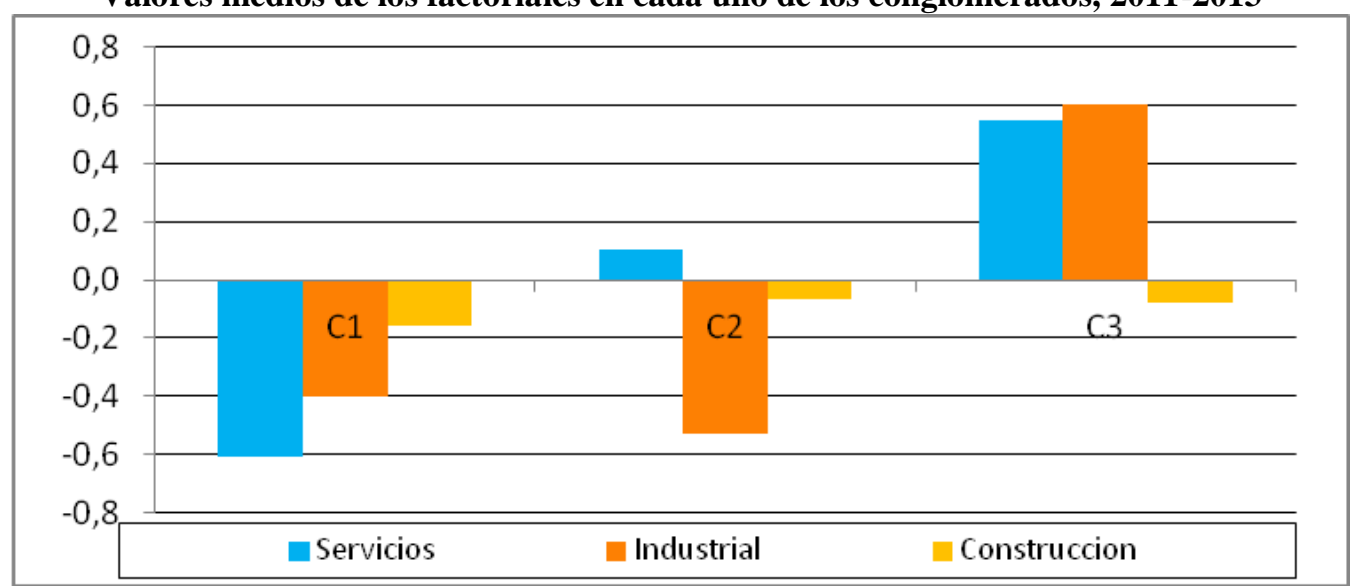

Fuente: Instituto de Estadística de Madrid, registros de desempleo. Los valores de 2013 se han estimado a partir de la población de 2012.

El segundo grupo (C2) tiene un carácter suburbano dentro de la RMB. Se encuentra concentrado en las zonas más periféricas y en hombres mayores de 45 años. El equivalente en la RMM, sería la presencia del conglomerado (C3) en las comarcas más alejadas. El tercer grupo (C3) de la RMB está relacionado con el aumento del desempleo entre extranjeros y trabajadores de categorías medio bajas, podría encontrar un paralelismo con el $\mathrm{C} 2$ de la RMM, que agrupa a municipios con aumentos intermedios. El cuarto grupo (C4) sería el vinculado a desempleo en colectivos socialmente vulnerables y a los factores de desempleo femenino, juvenil y de trabajadores mayores. En la RMM, podría equivaler al C3, aunque más alejados de la ciudad central. Finalmente, hay una serie de conglomerados atípicos, con valores extremos variados, pero asociables a fuerte aumento de las tasas de desempleo, especialmente en el sector de la construcción. 
Figura 21

Conglomerados de la evolución del desempleo en la RMB, 2011-2013

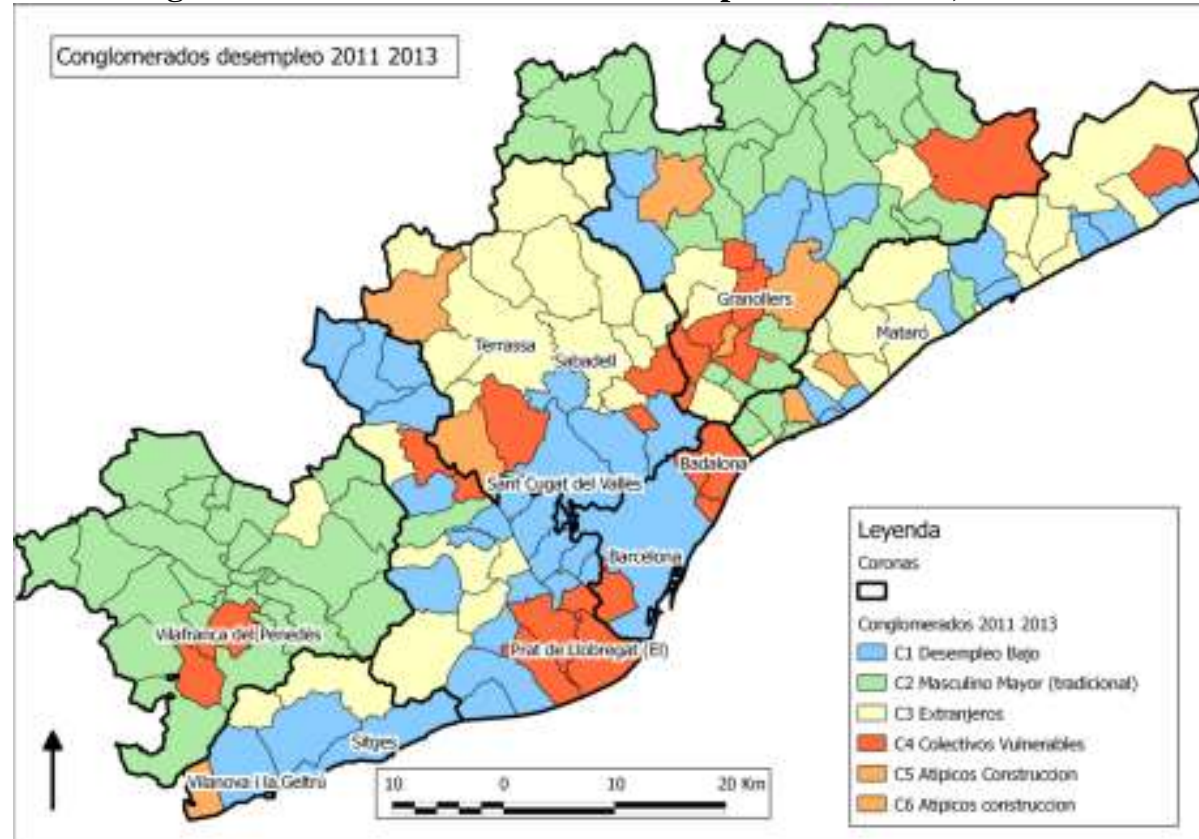

Fuente: Elaboración propia a partir de los registros de desempleo del Observatori Metropolità del Treball.

Figura 22

Conglomerados de la evolución del desempleo en la RMB, 2011-2013

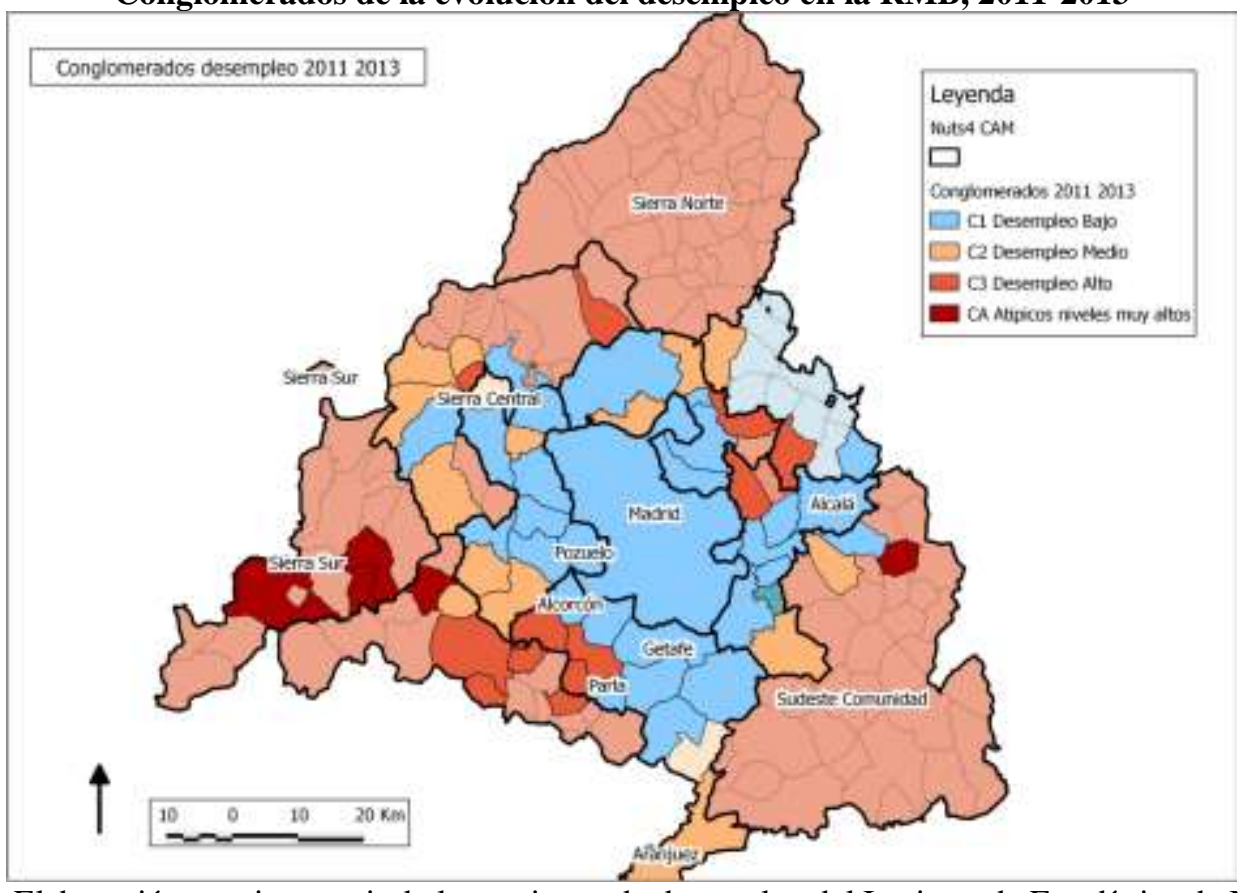

Fuente: Elaboración propia a partir de los registros de desempleo del Instituto de Estadística de Madrid. Los valores de 2013 se han estimado a partir de la población de 2012.

\section{Conclusiones}

El análisis territorial de 5 años de crisis (2008-2013) en las grandes regiones metropolitanas de Madrid y Barcelona permite establecer diferentes tipos de conclusiones: en clave de comparativa territorial, sobre las características específicas e 
internas de la crisis y un último grupo de reflexiones sobre los componentes territoriales de la crisis.

\section{Dinámicas territoriales}

La propia metodología comparativa territorial ofrece conclusiones independientes de la crisis pero relevantes de cara a los análisis comparados entre la RMM y la RMB. En primer lugar, las diferencias de escala y de reconfiguración administrativa. Barcelona sería equivalente a la zona central de Madrid y para comparar adecuadamente todo el municipio de Madrid, debe tomarse como referencia el conjunto del Barcelonès. Los municipios metropolitanos de clase alta sufren también una diferencia de escala, presentándose cierta equivalencia entre Sant Cugat del Vallès y los municipios del Oeste Metropolitano de Madrid. Finalmente hay una diferencia muy marcada entre el carácter monocéntrico de Madrid que ejerce un fuerte peso gravitatorio en todos los municipios (excepto quizás Alcalá de Henares) y el carácter pluricéntrico de Barcelona que genera mayores diferencias territoriales. Así, el territorio en torno a Madrid es más homogéneo, hasta el punto que la principal diferencia territorial durante el periodo 2011-2013 en la RMM sea la que distingue entre municipios de mayor y menor población y distancia de la capital.

\section{Características específicas}

Entre las conclusiones relativamente aterritoriales, cabe revisar la hipótesis que planteaba la existencia de dos fases muy diferentes, o dos crisis en una, en vez de una crisis continuada. Los resultados no son concluyentes para las dos regiones. Mientras que en la RMB sí que aparece una diferenciación muy grande entre una crisis sectorial durante el primer periodo y una general con componentes sociales durante el segundo; en la RMM, aparece un primer periodo también marcadamente sectorial y un segundo periodo relativamente inconcluyente, en el que se mezclan inercias del primer tipo de crisis junto a otros componentes. Quizás las medidas de reactivación de la actividad en general y de la actividad constructiva en particular que se activaron en 2010 (Plan E, entre otras) incidieron más en Madrid que en Barcelona. En todo caso, es muy posible que una ampliación del periodo de estudio y una actualización de los datos permitan confirmar totalmente la hipótesis de dos fases bien diferenciadas, desambiguando los datos de la segunda fase de la crisis en la RMM.

Por sectores, cabe destacar el conocido aumento del desempleo en la construcción a partir de 2008, que incluía también un aumento muy relacionado del desempleo industrial y subidas coincidentes en servicios y agricultura. Relacionado con este componente sectorial, aparece el protagonismo de la población de nacionalidad extranjera. Los extranjeros pasan de ser protagonistas en el desempleo por su asociación con el sector de la construcción a serlo por formar parte de los colectivos socialmente más vulnerables y de las categorías ocupacionales más bajas, que son quienes más sufren desempleo en la segunda parte de la crisis.

La crisis en general, pero más especialmente a partir de 2011, tiene un componente fuertemente social: incide especialmente en la población extranjera, en las mujeres, los trabajadores mayores, y los más jóvenes, más en las categorías profesionales más bajas y en los territorios más alejados y vulnerables. Se hace poco hincapié en la sustitución de los trabajadores mayores, con mayor categoría, formación, experiencia y mejores 
condiciones laborales. Esta sustitución supone un proceso general de reducción de costes y condiciones laborales que se hace visible en la renovación de las plantillas y que puede haberse visto potenciado por el Real Decreto ley 10/2010, de 16 de Junio que permitía reducir la cuantía de la indemnización por despido de 45 días (mayoritaria en los contratos laborales más antiguos) a 20. El acento mediático en el drama del desempleo juvenil, que siendo importante supone el $16 \%$ de la población desempleada $^{12}$, en ocasiones tiene el efecto indeseado de justificar las medidas dirigidas a conseguir más empleo a costa de la degradación de los salarios y las condiciones laborales cuando las políticas de empleo no deberían sacrificar ni la cantidad ni la calidad del empleo. Este tipo de aumento del desempleo sintetizaría varios procesos afines entre sí: reducción de costes laborales mediante erosión de las condiciones laborales y renovación y "rejuvenecimiento" de las plantillas, recortes en servicios públicos y protección social, desequilibrios socioterritoriales...

El componente social de la crisis plantea una dificultad a investigaciones como la presentada aquí y que tan sólo analiza el del desempleo. Una gran parte del impacto de la crisis en las poblaciones está relacionada con el deterioro de las condiciones laborales y de los salarios. Este deterioro no puede medirse (solo intuirse) empleando datos de desempleo, pero esta dificultad no niega su importancia como impacto y consecuencia de la crisis. Junto al desempleo, también se han resentido las condiciones laborales y salariales y su análisis resulta también fundamental para evaluar los impactos que se han producido. Además, de la misma forma que los efectos de la crisis no se pueden reducir al desempleo, la recuperación, en caso de producirse, no se puede evaluar adecuadamente sólo en función de la recuperación del empleo.

\section{Componentes territoriales de la crisis}

Tal y como se aventuraba en las hipótesis, se confirma que los territorios metropolitanos más afectados por la crisis son aquellos que se encuentran en posiciones subordinadas: bien en función de su ubicación periférica, bien a causa de las características socioeconómicas de su población residente y su mercado laboral local.

La evolución del desempleo ha pasado de una crisis que comienza en las zonas menos pobladas y alejadas de las ciudades centrales, a un proceso de degradación que es mayor en los territorios y colectivos cuya composición socioeconómica es de categoría menor. Las diferencias entre Madrid y Barcelona son notables: en la RMB se diferencia de forma muy clara un primer periodo de crisis marcado por el fin de la burbuja inmobiliaria y un segundo periodo marcado por un aumento del desempleo en los colectivos vulnerables; mientras que en la RMM parece alargarse la corrección de la burbuja inmobiliaria más, ya que presenta un primer periodo con menores aumentos que en Barcelona y un segundo periodo en el que el desempleo constructivo sigue teniendo un peso relevante.

Respecto a la posibilidad, anunciada en las hipótesis, de que las capitales globales estuvieran reforzando su posición respecto al resto del territorio nacional ${ }^{13}$, el análisis de la evolución de las tasas de desempleo, así como la aproximación multivariable que

\footnotetext{
${ }^{12}$ Rocha y Aragón, 2012.

${ }^{13}$ Graham y Marvin 2001.
} 
se ha llevado a cabo para determinar los componentes de la crisis no dejan lugar a dudas: efectivamente las capitales metropolitanas mejoran respecto al resto del territorio tanto en época de crecimiento, como en época de crisis. Este aumento de los desequilibrios territoriales entre capital, ciudades medias y entorno suburbano no responde sólo a una acumulación de funciones de las capitales. Por una parte, las capitales son menos dependientes de actividades relacionadas con la construcción y, por otro, muy posiblemente estemos asistiendo en paralelo a un proceso de cambio en la composición socioeconómica de la población de las capitales en la que la población de clase media y trabajadora del centro va dando da paso a una población de clase medio-alta y alta ${ }^{14}$. Este proceso se produce en Barcelona y en Madrid, aunque de forma menos evidente debido a la diferente evolución de la configuración administrativa de ambas capitales en un proceso similar al descrito para París ${ }^{15}$. Otras capitales pero parece producirse también en otras capitales europeas. Mientras que Madrid incorporó a su término municipal los municipios conurbanos de fuerte presencia obrera, Barcelona no lo hizo. Esta nueva población de mayor estatus socioeconómico se ve menos afectada por la crisis, su progresiva concentración territorial en el centro de las regiones metropolitanas ocasiona que éstas presenten un comportamiento aún mejor de lo esperable por el simple efecto de su composición sectorial del empleo. Este aumento de la condición socioeconómica de los centros urbanos de grandes capitales

Por resumir los elementos fundamentales del impacto territorial de la crisis, tendríamos que en primer lugar: existe un grupo de municipios vinculados a la ciudad central que por una combinación de posición en la jerarquía funcional de la RMB, concentración poblacional en categorías laborales altas y especialización sectorial ciertas ramas del sector servicios, mantienen crecimientos moderados y bajos del desempleo en las dos fases de la crisis analizadas. En segundo lugar, aparece un aumento de la relación entre desempleo y discriminación, especialmente en los municipios de menor nivel socioeconómico. Esta relación resulta más visible en la $\mathrm{RMB}$, aunque es de esperar que las cifras de los próximos años lo muestren también claramente en la RMM, ya que estos elementos aparecen ya presentes en algunos datos. En la RMB, comienza y termina antes el proceso de destrucción el empleo ligado a la burbuja inmobiliaria, ésta parece ser la clave de la evolución de la crisis: una situación de aumento generalizado de las tasas que es, a la vez, un proceso de acentuación de las desigualdades, también en su articulación territorial. Finalmente, aparece una pauta territorial y sectorial que localiza un último tipo de municipios que tienen buena evolución frente al desempleo en el periodo 2011-2013. Estaría formado por los municipios próximos a las concentraciones de población de clase alta y aquellos en los que existe especialización industrial.

\section{Bibliografía}

ALONSO, L.E. Centralidad del trabajo y cohesión social ¿Una relación necesaria? Gaceta sindical: reflexión y debate, Madrid: CCOO, 2006. p. 101-126, n. 7. ISSN: 1133-035X.

\footnotetext{
${ }^{14}$ López Gay, 2012.

${ }^{15}$ Clerval, 2010.
} 
CLERVAL, A. Les dynamiques spatiales de la gentrification à Paris. Cybergeo: European Journal of Geography, Espace, Société, Territoire, 2010 [En Línea] article 505, cybergeo.revues.org/23231.

GIL-ALONSO, F.; BAYONA, J. y VONO, D. Las migraciones internas de los latinoamericanos en España: del boom a la crisis econòmica. Papeles de Población, vol. 18, n. 71, 2012. p. 1-42.

GÓMEZ LEÓN, M. y MIRET, P. Caída de la actividad en la población adulta: ¿Un efecto de la crisis económica o de la estructura del mercado de trabajo?. En: La población en clave territorial. Procesos, estructuras y perspectivas de análisis. Actas del XIII Congreso de la Población Española. Santander: Ministerio de Economía y Competitividad, Gobierno de Cantabria, Asociación de Geógrafos Españoles y Universidad de Cantabria, 2012. p. 413-421.

GRAHAM, S. y MARVIN, S. Splintering urbanism: Networked infraestructures tecnological mobilities and the urban conditions. 2001. Londres, Inglaterra: Routledge.

LÓPEZ GAY, A. El regreso de la población a los centros metropolitanos españoles. Una visión demográfica y territorial de los procesos de reurbanización. Revista de la Facultad de Arquitectura Universidad Autónoma de Nuevo León, Nuevo León: UANL, 2012, p. 33-49, nº 6.ISSN 2007-1639.

ESTEBAN, J. La ordenación urbanística: conceptos, herramientas y prácticas, Barcelona: Diputación de Barcelona, 2003. ISBN: 84-8156-344-7.

RINKEN, S. El impacto de la crisis sobre la opinión pública ante la inmigración. En GUALDA CABALLERO, M. (ed.), Inmigración, ciudadanía y gestión de la diversidad, p. 221-243. Sevilla: Universidad Internacional de Andalucía, 2012.

ROCHA, F. y ARAGÓN, J. La crisis económica y sus efectos sobre el empleo en España. Gaceta sindical: reflexión y debate, vol. 19, 2012, p. 67-90.

RUBIALES, M., BAYONA, J. y PUJADAS, I. Patrones espaciales de la segregación residencial en la Región Metropolitana de Barcelona: Pautas de segregación voluntaria de los grupos altos. Scripta Nova, Revista Electrónica de Geografía y Ciencias Sociales. [En línea]. 2012. Barcelona: Universidad de Barcelona. Vol. XVI, núm. 423.

(c) Copyright Miguel Rubiales Pérez, 2016.

(C) Copyright Scripta Nova, 2016.

Ficha bibliográfica:

RUBIALES PÉREZ, Miguel. Territorio y crisis. Impacto territorial de la crisis económica en las regiones metropolitanas de Madrid y Barcelona. Scripta Nova. Revista Electrónica de Geografía y Ciencias Sociales. [En línea]. Barcelona: Universidad de Barcelona, 30 de noviembre de 2016, vol. XX, no 549-7. <http://www.ub.es/geocrit/sn/sn-549-7.pdf>. ISSN: $1138-9788$. 